\title{
Distributions of alpha particles escaping to the wall because of sawtooth oscillations in TFTR
}

\author{
Ya. I. Kolesnichenko* $\Gamma$ V. V. Lutsenko* $\Gamma$ R. B. White ${ }^{* *} \Gamma Y u$. V. Yakovenko* $\Gamma$ \\ and S. J. Zweben** \\ *Scientific Centre "Institute for Nuclear Research", Kyiv, 252650, Ukraine \\ **Princeton Plasma Physics Laboratory, P.O.Box 451, Princeton, NJ, 08543, USA
}

(October 29, 1998)

\begin{abstract}
It has been observed experimentally in deuterium-tritium shots of the Tokamak Fusion Test Reactor (TFTR) that crashes of sawtooth oscillations may result in very inhomogeneous flux of alpha particles to the wall. Namely, measurements with four detectors installed at the wall at $20^{\circ}, 45^{\circ}, 60^{\circ}$ and $90^{\circ}$ below the midplane of the torus have shown that the alpha flux to the wall is strongly peaked at the $20^{\circ}$ and $90^{\circ}$ detectors and on the noise level at the $45^{\circ}$ detector. To explain this phenomenon, both theoretical analysis and numerical simulation have been carried out. It is concluded that the "crash-induced prompt loss", i.e., the orbital loss of marginally trapped particles arising because of the crash-induced orbit transformation of circulating particles, is responsible for the flux to the $90^{\circ}$ and $60^{\circ}$ detectors, whereas the crash-induced stochastic diffusion of moderately trapped particles explains the large signal at the $20^{\circ}$ detector. The calculated poloidal distributions of the integral alpha flux are in reasonable agreement with experimental data. In addition to the integral flux, the flux of particles with given energy was calcu-
\end{abstract}


lated. The energy spectrum of the escaping particles has also been calculated, which can be used for diagnostics of the crash type. 


\section{INTRODUCTION}

Sawtooth oscillations are a typical form of the magnetohydrodynamic (MHD) activity in tokamak plasmas. They lead to oscillations of the profile shapes of the core plasma parameters within the so-called "sawtooth mixing region". Furthermore sawtooth oscillations can also essentially redistribute fast (superthermal) ions. The influence of sawteeth on fast ions with energies varying in a wide region $\Gamma$ from tens of kiloelectronvolts to several mega-

electronvolts $\Gamma$ has been studied both experimentally ${ }^{1-4}$ and theoretically $\mathrm{I}^{5^{-7}}$ and the main features of the fast ion transport have already been revealed. However $\Gamma$ till now all studies have been relevant mainly to the redistribution inside the sawtooth mixing region. Much less attention of researchers has been received by a related phenomenon - expulsion of fast ions from the mixing region in particular $\mathrm{t}$ to the wall. This phenomenon is addressed in the present work.

Investigation of the sawtooth-crash-induced escape of fast ions is of importance for better understanding of the physics of fast ion transport Tas well as for reliable modeling of the effect of sawtooth oscillations on the energy deposition of alpha particles and other fast ions in tokamak reactors. Moreover $\Gamma$ studying the lost particles $\Gamma$ one can make certain conclusion concerning the physics of the sawtooth crash itself.

The purpose of this work is to develop a theoretical approach to finding the fast ion flux to the wall caused by sawtooth oscillations and to report the results of experimental observations $\Gamma$ as well as of numerical modeling Tof alpha particles escaping to the wall in DT plasmas of the Tokamak Fusion Test Reactor (TFTR). ${ }^{8}$

The paper is organized as follows. The section II describes the experimental detecting of the alpha particle flux to the wall in the presence of sawtooth oscillations in DT shots in TFTR. The section III deals with numerical simulations of the sawtooth-crash-induced escape of single energetic particles. In particularГequations of the particle motion $\Gamma$ which take into account $\Gamma$ first $\Gamma$ temporal evolution of the magnetic configuration during a crash and $\Gamma$ 
second $\Gamma$ presence of the vacuum gap between the plasma and the first wall $\Gamma$ are formulated. These equations are applied to studying the crash-induced escape of various groups of alpha particles to the wall in experiments on TFTR. The section IV contains an analysis of the fluxes of energetic particles to the wall associated with two different physical mechanisms $\Gamma$ namely the crash-induced prompt loss (studied numerically in Sec. III) and the crash-induced ripple loss. This section presents also an analytical approach to finding the poloidal wall load distribution $\Gamma$ as well as the energy and pitch-angle distributions of fast ions $\Gamma$ which result from the crash-induced prompt loss. In Sec. VT this approach is used to calculate the distributions of the $\alpha$ particles escaping to the wall in TFTR and the obtained results are compared with experimental data. Finally results are contained in Sec. VI.

\section{EXPERIMENTAL OBSERVATIONS OF ALPHA LOSS DURING SAWTOOTH CRASHES IN TFTR}

Observations of the alpha loss during sawtooth crashes were made using the lost alpha scintillation detectors located $90^{\circ} \Gamma 60^{\circ} \Gamma 45^{\circ}$ Tand $20^{\circ}$ below the outer midplane of the vacuum chamber (in the ion $\nabla B$-drift direction). These detectors measured the alpha flux into small apertures $\left(\approx 0.01 \mathrm{~cm}^{2}\right)$ located near the wall of the TFTR vacuum chamber the plasma. The first three of these apertures were located just behind the geometrical shadow of the outer limiter $\mathrm{I}^{9}$ while the $20^{\circ}$ detector was on a radially movable probe which could be positioned within $\pm 2 \mathrm{~cm}$ of the outer limiter shadow. ${ }^{10}$ The total scintillator light emission vs. time for each of these detectors was measured with a bandwidth of up to $\approx 150 \mathrm{kHz}$ and digitized at a rate of up to $500 \mathrm{kHz}$.

Sawteeth do not normally occur in TFTR during high current Thigh power Neutral Beam Injection (NBI) heated discharges Tbut did occur late in time during discharges with lowered NBI power $(I=2.0 \mathrm{MA \Gamma R}=2.52 \mathrm{~m} \Gamma \approx 10 \mathrm{MW} \mathrm{NBI})$. Although these sawteeth did cause an 
internal redistribution of the confined alphas $\Gamma$ they did not cause any detectable alpha loss. ${ }^{4,11}$ However $\Gamma$ clear examples of sawtooth-induced alpha loss was observed during lower power $\Gamma$ lower current discharges created for another experiment Tas illustrated in Fig. 1 ( $I=1.4 \mathrm{MA} \Gamma$ $R=2.52 \mathrm{~m} \Gamma 7.5 \mathrm{MW} \mathrm{NBI})$. These sawteeth had a larger mixing radius than in the 2.0 MA discharges $\Gamma$ but had a similar $q(0) \approx 0.8$ and $q(a) \approx 5$.

A closer look at the time evolution of these low-current sawteeth is shown in Fig. $2 \Gamma$ where the alpha loss signals had a bandwidth of $\approx 150 \mathrm{kHz}$ and were digitized at $500 \mathrm{kHz}$. The duration of the sawtooth-induced increase in alpha loss was about the same as the time of the central temperature collapse of $\approx 50 \mu \mathrm{s} \Gamma$ but the time evolution was different for the three alpha detectors which saw a sawtooth-induced alpha loss. This is likely to be related to the complex space-time evolution of the sawtooth crash itselfГsome of which can be seen in the electron temperature signals shown in the figure.

The poloidal dependence of the magnitude of the sawtooth-induced alpha loss relative to the pre-sawtooth alpha loss is illustrated in Fig. 3. The rise in alpha loss is large at the bottom $90^{\circ}$ detector and at the midplane $20^{\circ}$ detector $\Gamma$ very small at the $60^{\circ}$ detector $\Gamma$ and not visible in the $45^{\circ}$ detector. The instantaneous alpha loss can increase by up to a factor of 10 during the crash but the time-integrated sawtooth-induced alpha loss is negligible with respect to the normal loss processes without the sawtooth $\Gamma$ i.e. $\Gamma$ first-orbit loss at the $90^{\circ}$ detector $^{9}$ and toroidal field ripple loss in the $20^{\circ}$ detector. $^{10}$

The behavior of alpha loss during these DT sawteeth is very similar to that measured earlier for DD fusion products loss during DD sawteeth in TFTR. ${ }^{12}$ The sawtooth-induced loss in the $90^{\circ}$ detector occurs mainly near the passing-trapped boundary and near the birth energy. It is interesting to note that not every sawtooth crash caused a burst of alpha loss $\Gamma$ as can be seen in Fig. 1; evidently the condition for sawtooth loss is sensitive to the details of the $q(r)$ profile or perhaps also the sawtooth crash. 


\section{NUMERICAL STUDY OF INFLUENCE OF SAWTOOTH CRASHES ON BEHAVIOR OF SINGLE ALPHA PARTICLES IN TFTR}

Let us assume that the pre-crash and post-crash states are axisymmetric. Then $\Gamma$ to describe the fast-ion motion during a sawtooth crash and immediately after it $\Gamma$ we can use the following equations for the particle coordinates $(\vec{r}) \Gamma$ energy $(\mathcal{E})$ and magnetic moment $(\mu):$

$$
\begin{gathered}
\dot{\vec{r}}=\vec{v}_{\|}+\vec{v}_{D}+\vec{v}_{E}, \\
\dot{\mathcal{E}}=\vec{E} \cdot\left(\vec{v}_{\|}+\vec{v}_{D}\right), \\
\dot{\mu}=0,
\end{gathered}
$$

where the dot denotes the time derivative along the guiding center orbit; $\vec{v}_{\|} \Gamma \vec{v}_{D} \Gamma$ and $\vec{v}_{E}$ are the velocities of the particle motion along the magnetic field $\Gamma$ of the toroidal drift $\Gamma$ and of the $\vec{E} \times \vec{B} \operatorname{drift} \Gamma$ respectively; $\vec{v}_{\|} \equiv v_{\|} \vec{B} / B ; v_{\|}= \pm\left\{(2 / M)\left[\mathcal{E}-\mu B_{0}(1-\epsilon \cos \vartheta)\right]\right\}^{1 / 2}$; $\vec{v}_{D} \equiv-\vec{v}_{\|} \times \nabla\left(v_{\|} / \omega_{B}\right) ; \vec{v}_{E} \equiv c \vec{E} \times \vec{B} /|B|^{2} ; \epsilon=r / R_{0} ;$ where $r$ is the radial coordinate such that $r=$ const at a flux surface $\Gamma \vartheta$ is the poloidal angle; $\vec{B}$ is the superposition of the equilibrium magnetic field and the magnetic field of the perturbation associated with the crash; $R_{0}$ is the radius of the magnetic axis; $B_{0}$ is the magnetic field at the axis; and $\vec{E}$ is the electric field.

Taking into account that sawteeth are localized in the plasma core and that $\Gamma$ in general $\Gamma$ there is a vacuum gap between the plasma edge and the chamber wall $\Gamma$ we determine in different ways the electromagnetic field in three regions: $0 \leq r \leq r_{m i x} \Gamma r_{m i x} \leq r \leq a$ and $r \geq a$ (in the vacuum gap) $\Gamma$ where $r_{m i x}$ is the sawtooth mixing radius $\Gamma$ and $a$ is the minor radius of the plasma.

In the sawtooth mixing region $\Gamma$ we describe the perturbed electromagnetic field in terms of the helical magnetic flux $\Psi$ $\Psi$ as follows: ${ }^{6}$ 


$$
\vec{B}=B^{3} \vec{e}_{3}+\vec{e}^{3} \times \nabla \Psi, \quad \vec{E}=\frac{1}{c} \frac{\partial \Psi}{\partial t} \vec{e}^{3}-\nabla \Phi
$$

Here $\vec{e}_{j}$ and $\vec{e}^{j}(j=1,2,3)$ are the base co- and contravariant vectors; the coordinate system $(r, \alpha \equiv \vartheta-\varphi, \varphi)$ is used; $\varphi$ is the toroidal angle; $B^{3}=B_{0} R_{0} / R^{2} ; R$ is the distance to the axis of the symmetry; and $\Phi(r, \alpha, t)$ is the electric potential related to $\Psi$ by the equation $\vec{E} \cdot \vec{B}=0 \Gamma$ which can be written in the form:

$$
\frac{\partial \Psi}{\partial t}+\frac{c R}{B \sqrt{g}}\left(\frac{\partial \Phi}{\partial r} \frac{\partial \Psi}{\partial \alpha}-\frac{\partial \Phi}{\partial \alpha} \frac{\partial \Psi}{\partial r}\right)=0,
$$

where $g$ is the determinant of the metric tensor $g_{i j}$. The equations (4) $\Gamma(5)$ imply that the sawtooth crash is associated with development of the $m=n=1$ helical perturbation and that the helical symmetry is conserved during the crash. They were derived in the assumption that the toroidal magnetic field is not perturbed by the crash. The temporal evolution of $\Psi$ for $r<r_{m i x}$ depends on the chosen model of the crash. In this work we will use the analytical expression for $\Psi(r, \alpha, t)$ that was suggested in Ref. ${ }^{6}$ and models the Kadomtsev-type crash. Note that a part of the electric field associated with perturbations plays the key role in redistribution of fast ions. ${ }^{6,15}$ On the other hand $\Gamma$ the equilibrium electric field $\Gamma E_{0}$ Tinfluences only the mode rotation. ${ }^{16}$ Therefore $\Gamma$ we take $E_{0}=0$.

In the region $r_{m i x} \leq r \leq a \Gamma$ we use Eq. (4) $\Gamma$ where $\Phi=0$ एand $\Psi$ does not depend on time and is expressed through the safety factor $\Gamma q(r) \Gamma$ as follows:

$$
\Psi=\int d r\left[q^{-1}(r)-1\right] \sqrt{g} B^{3}
$$

The equation (6) is also valid for $r<r_{\text {mix }}$ before and after the crash.

Finally flux $\Gamma \Psi_{p}$ :

$$
\vec{B}=\vec{B}_{T}+\nabla \varphi \times \nabla \Psi_{p}
$$

where $\vec{B}_{T}$ is the toroidal magnetic field $\Gamma B_{T}=R_{0} / R$. To find $\Psi_{p} \Gamma$ we use the toroidal component of the equation $\nabla \times \vec{B}=0$ given by: 


$$
R \frac{\partial}{\partial R}\left(\frac{1}{R} \frac{\partial \Psi_{p}}{\partial R}\right)+\frac{\partial^{2} \Psi_{p}}{\partial z^{2}}=0
$$

where $z$ is the vertical coordinate.

We assume that the plasma touches the wall near the inner midplane of the torus and that the vacuum gap is narrow $\Gamma 2\left(a_{c}-a\right) \ll a \Gamma$ where $a_{c}$ is the radius of the vacuum vessel cross-sectionTsee Fig. 4. Due to these assumptions $\Gamma$ we can suggest a model for description of the magnetic field in the vacuum region $\Gamma$ which $\Gamma$ first $\Gamma$ is rather simple and $\Gamma$ second $\Gamma$ does not require to know the shape of the cross-sections of the flux surfaces outside the vacuum vessel (the latter is important because the field lines that go through the vacuum gap intersect the wall and may form flux surfaces with complicated shapes or even produce no flux surfaces).

If the vacuum gap is very narrow $\Gamma$ it produces a negligible effect on the fast ion orbits at all poloidal angles except for small ones $\Gamma$ where flux surfaces can be approximated by Shafranov-shifted circles:

$$
r^{2}=z^{2}+\left[R-R_{0}+\Delta(r)\right]^{2}
$$

where $\Delta(r)$ is the Shafranov shift. Then it is sufficient to find a solution of Eq. (8) Гwhich well approximates $\Psi_{p}(R, z)$ only in the region of small $\vartheta$. In TFTR the region with circular flux surfaces is even wider $\Gamma$ ranging $\Gamma$ at least $\Gamma$ up to $|\vartheta|=\pi / 2$.

To obtain a solution of Eq. (8) in the mentioned part of the vacuum region $\Gamma$ we proceed from the variables $(R, z)$ to $(r, z)$. Then allowing for the fact that in the new variables $\Psi_{p}$ does not depend on $z \Gamma$ we take the equation at $z=0$. As a result $\Gamma$ we obtain the following equation:

$$
\Psi_{p}^{\prime \prime}+\frac{\Psi_{p}^{\prime}}{r}\left[\frac{R_{0}-\Delta}{R_{0}-\Delta+r}\left(1-\Delta^{\prime}\right)+\frac{r \Delta^{\prime \prime}}{1-\Delta^{\prime}}\right]=0 .
$$

where the primes mean radial derivatives.

One can show that when Eq. (10) is satisfied $\Gamma$ and the aspect ratio of the torus $(A)$ is large $\Gamma$ Eq. (8) in variables $(r, z)$ at $z \neq 0$ leads to the well-known equation for the Shafranov shift $\Gamma$ $\Delta .{ }^{14}$ However $\Gamma$ we will not use this equation because $A^{-1}$ is not a really small parameter 
in the region $r>a$. On the other hand $\Gamma$ the equation for $\Delta(r)$ for arbitrary $A$ is rather complicated. Therefore $\Gamma$ in order to specify $\Delta(r)$ Гwe expand it in a Fourier series $\Gamma$ restricting ourselves to the linear term:

$$
\Delta=\Delta_{a}+\Delta^{\prime}(r-a)
$$

where $\Delta_{a} \equiv \Delta(a)$ and $\Delta^{\prime} \equiv d \Delta /\left.d r\right|_{a}$ are parameters of our model. Substituting Eq. (11) into Eq. (10) Гwe find:

$$
\begin{aligned}
\Psi_{p}(r) & =\Psi_{p}(a)+\frac{B_{0} a^{2}}{q_{a}\left(R_{0}+\Delta_{a}+a\right)}\left(\frac{R_{0}+\Delta_{a}}{\Delta^{\prime}}-a\right)\left[\left(\frac{r}{a}\right)^{\Delta^{\prime}}-1\right] \\
& +\frac{B_{0} a^{3}}{q_{a}\left(R_{0}+\Delta_{a}+a\right)} \frac{1-\Delta^{\prime}}{1+\Delta^{\prime}}\left[\left(\frac{r}{a}\right)^{\Delta^{\prime}+1}-1\right]
\end{aligned}
$$

where $q_{a}=q(a)$.

It follows from Eq. (12) that $\Psi_{p}(r)$ depends on the Shafranov shift mainly through $\Delta^{\prime}$ (because $\Delta_{a} / R_{0} \sim A^{-2}$ ). We will take $\Delta_{a}=0$ (i.e. $\Gamma$ neglect the Shafranov shift within the plasma). To evaluate $\Delta^{\prime} \equiv d \Delta / d r$ Twe will use the expression given by: ${ }^{14}$

$$
\Delta^{\prime}=\frac{a}{R_{0}}\left[\frac{1}{\mathcal{J}^{2}(a)} \int_{0}^{a} d r \frac{\mathcal{J}^{2}(r)}{r}+\beta_{\mathcal{J}}\right] .
$$

where $\mathcal{J}(r)$ is the toroidal current inside the flux surface $r \Gamma \beta_{\mathcal{J}}$ is the ratio of the plasma pressure to the magnetic pressure of the toroidal current. When making a comparison of numerical results and experimental data $\Gamma$ we will vary $\Delta^{\prime} \Gamma$ which $\Gamma$ in fact $\Gamma$ will play the role of an adjustable parameter.

Based on the formulated equations Wwe studied the behavior of particles located inside the sawtooth mixing region before a crash. The results are shown in Figs. 5-9. The following parameters relevant to the TFTR DT shot \#87530 were used: $I=1.4 \mathrm{MA \Gamma} B_{0}=3.2 \mathrm{~T} \Gamma$ $a=87 \mathrm{~cm} \Gamma$ the minor radius of the wall $a_{c}=99 \mathrm{~cm} \Gamma$ the crash duration $\tau_{c r}=3.5 \times 10^{-5} \mathrm{~s} \Gamma$ $r_{m i x}=57 \mathrm{~cm} \Gamma q_{0} \equiv q(0)=0.8$. The profile of the inverse safety factor $\left(q^{-1}\right)$ was taken as two parabolas at $0 \leq r \leq r_{\text {mix }}$ and $r_{\text {mix }} \leq a$ Гaligned smoothly at $r=r_{m i x}$. In addition $\Gamma$ the $q=1$ radius was assumed to be $r_{s}=r_{m i x} / \sqrt{2}$. 
The figure 5 shows the effect of the sawtooth crash on particles with different $\lambda \Gamma$ which are located in the vicinity of the plasma center before the crash. To demonstrate the effect of the Shafranov shift $\Gamma$ two cases are presented: $\Delta^{\prime}=2 A^{-1} \Gamma$ which is close to the magnitude predicted by Eq. (13) $\Gamma$ and $\Delta^{\prime}=0$. It follows from Fig. 5 that marginally circulating particles $(\lambda=0.74-0.788)$ can be transformed during a crash into trapped ones $\Gamma$ which escape from the plasma the point where particles reaches the wall being dependent on $\lambda$. One can see that the trajectories in the vacuum gap essentially depend on $\Delta^{\prime}$. The presence of the Shafranov shift drastically changes the orbits of those particles which enter the gap at $|\vartheta| \lesssim 90^{\circ}$. Because of this $\Gamma$ particles can strike the wall only at $|\theta| \geq \theta_{\tau} \Gamma$ where $\theta$ is the poloidal angle at the wall $\Gamma \theta_{\tau}$ is determined by the orbit tangent to the wall.

The figure 6 shows orbits of escaping particles $\Gamma$ which have the same initial pitch angle and radial position but different energies. It follows from this figure that the sawtooth crash expels only particles with sufficiently high energy $\Gamma \mathcal{E}>\mathcal{E}_{\min } \Gamma$ where $\mathcal{E}_{\min }$ is the energy of the particle having the orbit tangent to the wall. In the considered example $\mathcal{E}_{\text {min }}=2.5 \mathrm{MeV \Gamma}$ but $\Gamma$ in general $\Gamma \mathcal{E}_{\text {min }}$ depends on the initial coordinates and $\lambda$. A general conclusion from Fig. 6 and 5 is that a particle can strike the wall $\Gamma$ i.e. $\Gamma$ the fast ion flux to the wall $(\Gamma)$ is not vanishing $\Gamma$ only for $\theta_{\min } \leq|\theta| \leq \theta_{\max } \Gamma$ where $\theta_{\min }=\min \theta_{\tau}(\mathcal{E})$. The existence of $\theta_{\max }$ is associated with the fact that the escaping particles strike the wall at $R>R_{t}\left(R_{t}\right.$ is $R$ at the turning point). Note that $\Gamma$ in general $\Gamma \theta_{\min }=\min \theta_{\tau}(\mathcal{E}, \lambda) \Gamma$ but $\Gamma$ in the case when only marginally trapped particles contribute to the $\operatorname{loss} \Gamma \theta_{\tau}$ does not depend on $\lambda \Gamma$ see Sec. IV.

The orbits of all observed escaping particles intersect the wall escape at $|\theta|=\theta_{\tau}$. Note that $\Gamma$ in contrast to this $\Gamma$ the orbits of all alpha particles lost due to collisional diffusion are tangent to the wall. ${ }^{13}$ The existence of $\theta_{\tau}$ is associated with the fact that the curvature radius of the orbits of marginally trapped particles near the wall at $\theta=0$ exceeds the radius of the vacuum vessel (therefore $\Gamma$ if a marginally trapped particle were produced from a circulating one byГe.g. Tthe pitch-angle scattering $\Gamma i t$ would never strike the wall at $|\theta|<\theta_{\tau}$ Tunlike what was claimed in Ref. $\left.{ }^{13}\right)$. A general condition providing existence 
of $\theta_{\tau}$ will be obtained later $\Gamma$ in Sec. IV.

The figures 7 and 5 (d) show the trajectories of two particles having the same initial values of $\lambda$ and $\mathcal{E}$ and starting from the same initial unperturbed orbit. We conclude from here that a particle may either escape or remain confinedГdepending on the initial poloidal angle $\Gamma$ i.e. $\Gamma$ on the phase of the perturbation. The same effect was observed for variation of the initial toroidal angle of a particle.

The figure 8 shows the effect of the crash on particles with various initial radial positions. We observe that only the particles that are sufficiently close to the plasma center before the crash can escape. The particles initially located in the vicinity of the mixing radius remain confined and may even approach the plasma center.

FinallyГFig. 9 demonstrates the possibility that trapped particles may also escape to the wall. However $\Gamma$ such a process is not typical because it can lead to escaping of very narrow group of particles with parameters close to the loss cone region.

\section{ANALYTICAL DESCRIPTION OF THE CRASH-INDUCED FLUX OF FAST IONS TO THE WALL}

\section{A. Wall areas irradiated by fast ions due to various physical mechanisms}

The energetic particle wall load is never homogeneous. Certain conclusions concerning the poloidal distribution of this load can be made by analyzing the physical mechanisms leading to the particle loss.

The simplest mechanism is associated with the finite particle orbit width $\Gamma$ which results in prompt loss with a non-vanishing flux to the wall either below or above (depending on the direction of the toroidal drift) the equatorial plane of the torus. It is clear that if a half of the wall (lower or upper) were absent $\Gamma$ the other part would be affected by particles. Therefore one can say that in the case of prompt loss $\Gamma$ a half of the wall surface is shadowed by another one. 
The effect of plasma MHD activity on fast ions is more complicated because it involves Tat least $\Gamma$ two processes. One of them is the interaction of fast ions with the MHD perturbation which expels particles from the plasma core. This process triggers one or more other ones leading to escape of particles to the wall. Such secondary processes can be various $\Gamma$ but typically only one or two $\Gamma$ namely $\Gamma$ the orbit transformation and stochastic diffusion $\Gamma$ are dominant. In particular $\Gamma$ circulating particles can be lost because of transformation into marginally trapped particles lying inside the loss-cone region. Such particles $\Gamma$ after the transformation occurs $\Gamma$ are lost within one bounce. This was observed in the examples considered in Sec. III. On the other hand trapped particles can be lost after the MHD perturbations expel them into the region of stochastic diffusion. Then particles are lost for many bounce periods (e.g. Tseveral tens) but quick enough for their confinement time to be much less than the slowing down time. In general $\Gamma$ the fluxes of particles associated with the diffusion and the orbit transformation have maxima at different magnitudes of the poloidal angle: the first one is more close to the midplane of the torus. Moreover Tbelow we will show that in the presence of the vacuum gap $\Gamma$ the wall areas affected due to the orbit transformation and the diffusion may even not overlap. The reason for this is that the gap produces an additional shadowing effect which is different for different groups of particles.

In Sec. III we found numerically that there is an angle $\theta_{\tau}$ such that marginally trapped particles after orbit transformation always strike the wall at $|\theta|>\theta_{\tau}$. This means that the vacuum gap produces an additional shadow in the region $-\theta_{\tau}<\theta<0$ for such particles (the toroidal drift here and below is assumed to be directed downwards). Now our aim is to show that $\Gamma$ in general $\Gamma$ the angle of the the shadow boundary $\Gamma \theta_{\tau} \Gamma$ exists for any particles and depends only on $\mathcal{E}$ and $\lambda$ at the moment when a particle strike the wall. This statement implies that the specific mechanism which provides the particle transport inside a plasma plays no role. The only restriction is that the perturbation of the particle orbit for the last bounce before escaping to the wall is negligible $\mathrm{bbut}$ it seems that this condition is satisfied in most cases of practical importance. Therefore our analysis is applicable $\Gamma$ in particularГto 
the case when the alpha flux is produced by the collisional diffusion $\Gamma$ when $\Gamma$ as it was shown in Ref. ${ }^{13}$ Tthe vacuum gap produces an additional shadow.

To find $\theta_{\tau} \Gamma$ we consider the particle orbit equation resulting from conservation of the canonical angular momentum $\Gamma$

$$
J=-\frac{e}{c} \Psi+M v_{\|} R=\text { const. }
$$

A sketch of particle orbits near the chamber wall is given in Fig. 10 Where we have schematically drawn a set of curves $J(r, \vartheta)=$ const corresponding to certain values of $\lambda$ and $\mathcal{E}$. One can see that only the parts that of the curves drawn by solid lines represent actual particle orbits. Therefore $\Gamma$ the part of the wall marked as $B C$ is shadowed. This part is characterized by decrease of $J$ with $\theta \Gamma \partial J / \partial \theta<0$ (for definiteness $\Gamma$ we consider here only the case of $v_{\|}>0 \Gamma$ which we are interested in). The point $B$ separating the shadowed and non-shadowed parts of the wall corresponds to a maximum of $J$ so that $\partial J /\left.\partial \theta\right|_{B}=0$. The last equation determines $\theta_{\min }$.

Differentiating $J$ over $\theta$ along the wall and using the equation

$$
r^{2}=a_{c}^{2}+\left[\Delta(r)+\Delta_{c}\right]^{2}+2 a_{c}\left[\Delta(r)+\Delta_{c}\right] \cos (\theta)
$$

which follows from the wall geometryГand Eq. (9)Гwe obtain:

$$
\frac{\partial J}{\partial \theta}=-M a_{c} v \sin (\theta)\left(\frac{1+\chi_{w}^{2}}{2 \chi_{w}}-\mathcal{G}\right)
$$

where $\chi_{w}=\left(1-\lambda / h_{w}\right)^{1 / 2}$ is the pitch-angle cosine at the wall; $h_{w}=\left[R_{c}+a_{c} \cos (\theta)\right] / R_{0}$; $a_{c}$ and $R_{c}$ are the minor radius of the vacuum vessel and the major radius of its center $\Gamma$ respectively;

$$
\mathcal{G}(\theta, \mathcal{E})=\left.\frac{1}{\rho B_{0}} \frac{\Delta+\Delta_{c}}{r-\Delta^{\prime}\left[\Delta+\Delta_{c}+a_{c} \cos (\theta)\right]} \frac{d \Psi_{p}}{d r}\right|_{r=r_{w}(\theta)}
$$

$r_{w}(\theta)$ is the value of $r$ at the wall at the poloidal coordinate $\theta \Gamma \Delta_{c}$ is the shift of the plasma center with respect to the chamber center. The quantity $\mathcal{G}$ represents the effect of the vacuum 
gap. When $\mathcal{G}=0$ (there is no vacuum gap) $\Gamma \partial J / \partial \theta$ is always positive for $-\pi<\theta<0$; therefore $\Gamma$ particles with any pitch angle can strike the wall at any point below the midplane of the torus. In the case when a particle with energy $\mathcal{E}$ strikes the wall at the point $\theta$ and $\mathcal{G}(\theta, \mathcal{E}) \leq 1 \Gamma$ there is no restriction on the particle pitch angle. When $\mathcal{G}(\theta, \mathcal{E})>1 \Gamma$ Eq. $(16)$ imposes the following restrictions on the pitch angle and $\lambda$ of the particles striking the wall at the point $\theta$ :

$$
\begin{gathered}
\chi_{w} \leq \chi_{\max }(\theta, \mathcal{E}) \equiv \mathcal{G}-\left(\mathcal{G}^{2}-1\right)^{1 / 2} \\
\lambda \geq \lambda_{\min }(\theta, \mathcal{E}) \equiv\left(1-\chi_{\max }^{2}\right) h_{w} .
\end{gathered}
$$

where $\chi_{\max }$ and $\lambda_{\min }$ correspond to the trajectory that is tangent to the wall $\partial J / \partial \theta=0$; and the equation $\lambda=\lambda_{\min }(\theta, \mathcal{E})$ determines $\theta_{\tau}(\mathcal{E}, \lambda)$.

If $\lambda<\lambda_{\min }(\theta, \mathcal{E})$ then the point $\theta$ is "shadowed" for the particles with given $\lambda$ and $\mathcal{E}$. It follows from Eq. (19) that the shadow decreases with $\lambda$. As $\mathcal{G}$ decreases with $\rho$ Tthe shadow also shrinks with increase of $\mathcal{E}$.

To demonstrate the different gap shadowing of various groups of escaping energetic particles $\Gamma$ we have calculated $\theta_{\tau}$ as a function of $\lambda$ for various magnitudes of $\mathcal{E}$ for the TFTR shot \#87530 at $t=4.45 \mathrm{~s}\left(\Delta^{\prime}=1.67 A^{-1}\right)$. The results are shown in Fig. $11 \Gamma$ where we have also taken into account the effect of the finite Larmor radius (i.e. Tthe difference between the particle position and the guiding center position) Wwhich is disregarded in Eqs. (16) and (17) [the Larmor corrections to these equations are given in Appendix AГsee Eqs. (A3) and (A4)]. However $\Gamma$ it turns out that the difference between $\theta_{\tau}$ shown in Fig. 11 and $\theta_{\tau}$ calculated from Eqs. (16)-(19) is negligible. This result is somewhat unexpected for the Larmor radius of the considered particles is not small $(4-10 \mathrm{~cm})$.

It follows from Fig. 11 that the particles escaping due to orbit transformation $(\lambda \approx 0.8)$ irradiate the wall at $|\theta|>30^{\circ}$. On the other hand $\Gamma$ one can expect that most particles escaping because of stochastic diffusion will have $\lambda$ exceeding unity (for such particles $\Gamma$ the 
banana tips are located in the region $|\vartheta|<\pi / 2$ एwhere the ripple is essential) and the energy close to $3.5 \mathrm{MeV}$. If we take $\Gamma$ e.g. $\Gamma \lambda=1.1$ and $\mathcal{E} \geq 2.5 \mathrm{MeV} \Gamma$ we find that the wall will be irradiated at $0^{\circ}<|\theta|<30^{\circ}$. Note that the $\lambda=1.1$ particles with $\mathcal{E}=3.5 \mathrm{MeV}$ and $\mathcal{E}=2.8 \mathrm{MeV}$ strike the wall at $\theta \approx 0^{\circ}$ and $\theta \approx 30^{\circ}$ Trespectively.

We can conclude from this analysis that $\Gamma$ in the considered exampleГthe particles escaping due to orbit transformation and stochastic diffusion irradiate different parts of the wall.

\section{B. General expressions for the prompt loss of fast ions induced by core-localized MHD perturbations}

A general conclusion from Sec. III and Subsec. IV A is that when the magnetic ripple is sufficiently weak in the region of sawtooth oscillations $\Gamma$ the dominant fraction of energetic particles striking the wall not too close to the equatorial plane of the torus consists of the particles that are marginally counter-circulating and localized near the magnetic axis before

the crash. These particle escape due to transformation into marginally trapped ones during the crash $\Gamma$ which is accompanied by sharp increase of the particle orbit width. We will refer to the loss of these particles as "the prompt loss induced by the crash". Below we restrict ourselves to the consideration of such loss $\Gamma$ assuming that only MHD perturbations break the axial symmetry of the magnetic field.

We distinguish two stages in the particle motion: first $\Gamma$ transport of the particle to the loss-cone region due to the crash; second Tmotion to the wall inside the loss-cone region. We assume that the process of the particle transport to the loss-cone region is known and that the effect of MHD perturbations on the particle motion after the orbit transformation is negligibly small. One can see that the latter assumption is justified in two limit cases $\Gamma$ which we will refer to as the "slow crash" and "quick crash" cases: (i) $\tau_{c r} \gg \tau_{b}\left(\tau_{b}\right.$ is the particle bounce/transit period); (ii) $\tau_{c r} \ll \tau_{b}$. Due to these assumptions $\Gamma$ we can write the following expression for the number of the lost particles: 


$$
N=\int_{L C R} d^{3} \vec{x} d^{3} \vec{v} G=\frac{4 \pi^{2} R_{0}}{M^{2}} \sum_{\sigma} \int_{L C R} d r d \vartheta d \mathcal{E} d \lambda r \frac{\mathcal{E}}{\left|v_{\|}\right|} G,
$$

where $L C R$ means the loss-cone region $\Gamma G$ represents the source of the ions in the loss-cone region. These particles produce non-uniform wall load. To describe it $\Gamma$ we define the particle flux to the wallГ $\Gamma(\theta, \mathcal{E}) \Gamma$ as the number of particles with given energy striking a unit surface of the wall for the crash time. This flux is associated with the number of lost particles as follows:

$$
N=\int d S d \mathcal{E} \Gamma(\theta, \mathcal{E})
$$

where $d S$ means integration over the wall surface. It is clear that the total flux density is $\int d \mathcal{E} \Gamma(\theta, \mathcal{E})$. Note that the introduced particle flux implies that we are not interested in the temporal evolution of the wall load during the crash.

Now we take advantage of the fact that the canonical angular momentum is conserved in the loss-cone region as MHD perturbations are neglected at this stage of the particle motion. Then we can express $\lambda$ through $\theta$ and $\Gamma$ using Eqs. (20) and (21) $\Gamma$ obtain the expression for $\Gamma(\theta, \mathcal{E})$ in the form:

$$
\Gamma=\frac{2 \pi}{M^{2}} \frac{1}{a_{c} h_{w}} \int d r d \vartheta\left|\frac{\partial \lambda}{\partial \theta}\right| \frac{r \mathcal{E}}{\left|v_{\|}\right|} G .
$$

It may be of interest to compare the crash-induced loss of fusion-produced alpha particles and their prompt loss. One can see that the prompt loss results in the flux of alphas to the wall for the crash time $\Gamma$ which can be determined by Eq. (22) with $G=n_{D} n_{T}\langle\sigma v\rangle \tau_{c r}$. Due to this $\Gamma$ we arrive at the following expression for the ratio $\Gamma / \Gamma_{\text {prompt }}$ :

$$
\frac{\Gamma}{\Gamma_{\text {prompt }}}=\frac{\int_{L C R} d r d \vartheta r \frac{\mathcal{E}}{\left|v_{\|}\right|}\left|\frac{\partial \lambda}{\partial \theta}\right| G}{\int_{L C R} d r d \vartheta r \frac{\mathcal{E}}{\left|v_{\|}\right|}\left|\frac{\partial \lambda}{\partial \theta}\right| n_{D} n_{T}\langle\sigma v\rangle} .
$$

The source function $G$ depends on the character of the plasma motion during the $\operatorname{crash} \Gamma$ as well as on the ratio $\tau_{b} / \tau_{c r}$. Below we consider the slow crash $\left(\tau_{c r} \gg \tau_{b}\right) \Gamma$ which seems to be of the largest practical importance. 
Note that the derived expressions can be used for description not only of the sawtoothinduced loss of fast ions but also of the loss produced by other burst-like core-localized MHD perturbations $\Gamma$ such as minor and major disruptions. Moreover $\Gamma$ one may expect that they are applicable also to the case of long lasting MHD perturbations $\Gamma$ in which case $N$ and $\Gamma$ are related to the unit time. However $\Gamma$ the expressions are restricted to those experiments where the main mechanism of escape of particles to the wall is prompt loss following the stage of interaction of particles with MHD perturbations (i.e. Tdiffusion is negligible).

\section{Prompt loss due to slow crash}

To use the expressions of previous subsection one should determine $G$ and calculate $\partial \lambda / \partial \theta$. This will be done below for the case of slow crash.

When $\tau_{b} \ll \tau_{c r} \Gamma$ a particle orbit weakly changes for a transit period. Therefore $\Gamma$ after the transformation the particle always passes in the vicinity of the $\mathrm{X}$ point of the separatrix between trapped and circulating orbits $\Gamma$ and $\Gamma$ thus $\Gamma$ the $\mathrm{X}$ point can be considered as a starting point of the particle motion at the second stage. This gives us grounds to assume that $G$ is proportional to $\delta(\vartheta-\pi) \delta\left(r-r_{X}\right) \Gamma$ where $r_{X}(\lambda, \mathcal{E})$ is the radial coordinate of the X point. On the other hand $\Gamma$ we obtain from Eq. (21) that

$$
d N=d^{3} \vec{v} \int_{L C R} d^{3} \vec{x} G .
$$

It follows from this equation that $\int_{L C R} d^{3} \vec{x} G$ is the number of fast ions with given $\mathcal{E}$ and $\lambda$ produced by the crash (or another MHD perturbation) in the loss-cone region in a unit volume of the velocity space. Therefore $\Gamma$ it can be presented as:

$$
\int_{L C R} d^{3} \vec{x} G=V_{X}(\lambda, \mathcal{E}) f(\lambda, \mathcal{E}),
$$

where $f$ and $V_{X}$ are the distribution function of particles and their effective volume entering the loss-cone region. Taking this into account $\Gamma$ we can write $G$ in the form: 


$$
G=\frac{f V_{X}}{2 \pi R_{0} r_{X} h_{X}} \delta\left(r-r_{X}\right) \delta(\vartheta-\pi)
$$

where $r_{X}$ is determined by the equations $\vartheta=\pi \Gamma \dot{\vartheta}=0$. Using Eq. (26) $\Gamma$ we can calculate the integrals in Eq. (22). This yields:

$$
\Gamma=\frac{\mathcal{E} f}{M^{2} R_{0} h_{w}} \frac{V_{X}}{\left|v_{\| X}\right|}\left|\frac{\partial \lambda}{\partial \theta}\right|
$$

Let us express $V_{X}$ through the plasma volume $\Gamma V_{0} \Gamma$ that moves since the beginning of the crash and reaches the radius $r_{X}(\lambda, \mathcal{E})$. To do this $\Gamma$ we have to make several assumptions. First $\Gamma$ plasma is assumed incompressible. Second $\Gamma$ we assume that only the particles which were located sufficiently close to the plasma center $\Gamma$ so that the initial longitudinal velocity can be approximated as $v_{\| 0} \approx v \sqrt{1-\lambda}$ Treach the loss-cone region during the crash. At last $\Gamma$ we assume that the particle energy and the magnetic moment are conserved and that the process of redistribution of circulating particles by MHD perturbations does not depend on $\lambda$ and $\mathcal{E}$. When making these assumptions $\Gamma$ we were motivated by the fact that circulating particles closely follow evolving flux surfaces due to relatively small radial width of the unperturbed orbits and slow toroidal precession. ${ }^{6,15}$ Then we can write:

$$
d N=f V_{0} d^{3} \vec{v}_{0}
$$

where

$$
d^{3} \vec{v}_{0}=\frac{\pi v^{2}}{M v_{\| 0}} d \mathcal{E} d \lambda=\frac{\pi v}{M(1-\lambda)^{1 / 2}} d \mathcal{E} d \lambda,
$$

Comparing Eqs. (24)Г(25) with Eqs. (28)Г(29) we find:

$$
V_{X}=V_{0} \frac{v_{\| X}}{v_{\| 0}}
$$

It follows from Eq. (30) that $V_{X} \ll V_{0}$. This is a consequence of the fact that the particles moving near the $\mathrm{X}$ point strongly slow down their guiding-center motion.

Due to Eq. (30) $\Gamma$ the flux of fast ions given by Eq. (27) is expressed through $V_{0}\left(r_{X}\right)$. The volume $V_{0}\left(r_{X}\right)$ can be calculated if the crash model is specified. It is worth to discuss 
some limitations of this approach. Its basic assumptions (circulating fast ions follow the flux surfaces; the ion energy is conserved) are fulfilled only approximately for $3.5-\mathrm{MeV} \alpha$ particles in TFTR especially when they approach the transformation point. This may explain why the particle behavior during the crash depends on the initial phase and radius (see Figs. 7 and 8). However the concept of $V_{0}\left(r_{X}\right)$ is convenient as it enables us to relates the flux of escaping fast ions to the motion of the thermal plasma during the crash. Below we find $V_{0}\left(r_{X}\right)$ for the Kadomtsev model.

For the sake of simplicity $\mathrm{\Gamma we}$ assume that the profile of $q^{-1}(r)$ is approximately parabolic. Then solving the equations of the flux conservation (e.g. Eqqs. (1) and (2) of Ref. ${ }^{17}$ ) Tone can show that the post-crash plasma layer with the radial coordinate $r^{+}$is a mixture of two pre-crash layers that were located before the crash at the radii $r_{1}^{-}=\left\{\left[r_{m i x}^{2}-\left(r^{+}\right)^{2}\right] / 2\right\}^{1 / 2}$ and $r_{2}^{-}=\left\{\left[r_{\text {mix }}^{2}+\left(r^{+}\right)^{2}\right] / 2\right\}^{1 / 2}$. One can see from these formulae that the volume of the plasma transported due to the crash outwards through the flux surface $r$ is

$$
V(r)=\pi^{2} R_{0} \max \left[0, \min \left(r_{m i x}^{2}-r^{2}, 2 r^{2}\right)\right]
$$

In order to take into account the radial excursions of the fast ion orbits from the bounceaveraged radial coordinate $\Gamma$ we take $V_{0}\left(r_{X}\right)$ to be slightly different from $V\left(r_{X}\right)$ Tnamely:

$$
V_{0}\left(r_{X}\right)=\pi^{2} R_{0} \max \left\{0, \min \left[r_{m i x}^{2}-\left(r_{X}-s q_{X} \rho\right)^{2}, 2\left(r_{X}-s q_{X} \rho\right)^{2}\right]\right\}
$$

where $s$ is a dimensionless quotient (the results presented below are obtained for $s=1$ ).

Note that it is assumed in Eq. (32) that the volume of the plasma reaching a certain radius $r=r^{\prime}$ from inside throughout the crash is the same as the volume of the plasma transported due to the crash outside this radius [given by Eq. (31)]. Of course Tthe dynamics of the plasma during a crash is more complicated. Some elements of the plasma volume may "visit" the region $r \geq r^{\prime}$ but be located at $r<r^{\prime}$ after the crash is over. Such elements will contribute to the fast ion transformation but are not taken into account by Eq. (32). Nevertheless $\Gamma$ bearing in mind all other sources of uncertaintyГEq. (32) seems to be a reasonable estimate for the amount of fast ions supplied to the radius $r_{X}$ by a Kadomtsev-type crash. 
Sensitivity of the results to the choice of $V\left(r_{X}\right)$ will be discussed in Sec. V.

To calculate $\partial \lambda / \partial \theta \Gamma$ we proceed from the following equation of conservation of the canonical angular momentum after the transformation:

$$
\frac{e}{m c}\left[\Psi_{p}(r(\theta))-\Psi_{p}\left(r_{X}\right)\right]=\left(v_{\| w} h_{w}-v_{\| X} h_{X}\right) R_{0}
$$

where $r(\theta)$ is the radius of the flux surface crossing the wall at the angle $\theta$; the superscripts ' $X$ ' and ' $w$ ' refer to the $\mathrm{X}$ point and the point where the particle strikes the wall; and $h=R / R_{0}$. As $\dot{\vartheta}=0$ at the $\mathrm{X}$ point $\Gamma$ we obtain:

$$
v_{\| X}=-\frac{q_{X} R_{0} v_{D X}}{r_{X}} \approx \frac{q_{X} v^{2}}{2 \omega_{B 0} r_{X}}
$$

where we have assumed that $v_{\| X} \ll v$. The equations $(34) \Gamma$

$$
\lambda=h_{X}\left[1-\left(\frac{v_{\| X}}{v}\right)^{2}\right]
$$

and

$$
v_{\| w}=h_{w}^{-1 / 2}\left(h_{w}-\lambda\right)^{1 / 2} v
$$

enable us to exclude $v_{\| X} \Gamma \lambda$ and $v_{\| w}$ from Eq. (33). Then Eq. (33) determines $r_{X}$ as a function of $\theta$ and $\mathcal{E}$ Talthough it seems difficult to solve it for $r_{X}$ explicitly. Differentiating Eqs. (33) and (35) at fixed energy $\Gamma$ excluding $d r_{X}$ Tand neglecting the terms $\sim \rho^{2} \Gamma$ we obtain:

$$
\frac{\partial \lambda}{\partial \theta}=-\sin (\theta) \frac{q_{X} \rho a_{c}}{r_{X} R_{0}}\left(1+\frac{q_{X} \rho}{2 r_{X} \chi_{w}}\right)^{-1}\left[\frac{1+\chi_{w}^{2}}{2 \chi_{w}}-\mathcal{G}(\theta, \mathcal{E})\right],
$$

where $\mathcal{G}(\theta, \mathcal{E})$ is given by Eq. (17). The second term of $\partial \lambda / \partial \theta$ represents the effect of the Shafranov shift. When this term is sufficiently large $\Gamma \partial \lambda / \partial \theta$ may change the sign. In our calculations we have observed both the case when $\partial \lambda / \partial \theta>0$ everywhere and the case when $\lambda(\theta)$ reaches maximum at $\theta=-\theta_{\tau}$. In the latter case $\Gamma$ the poloidal angle $\theta=-\theta_{\tau}$ corresponds to the trajectory tangent to the wall so that the particles cannot strike the wall at $|\theta|<\theta_{\tau}$ (see Sec. III and Subsec. IV A). 
Taking this into account $\Gamma$ we obtain from Eqs. (27) $Г(30)$ Tand (37):

$$
\begin{aligned}
\Gamma(\theta, \mathcal{E}) & =-\sin (\theta) \frac{f v^{2} q_{X} \rho V\left(r_{X}\right)}{2 r_{X} R_{0}^{2}(1-\lambda)^{1 / 2} h_{w}}\left(1+\frac{q_{X} \rho}{2 r_{X} \chi_{w}}\right)^{-1} \\
& \times \max \left\{\left[\frac{1+\chi_{w}^{2}}{2 \chi_{w}}-\mathcal{G}(\theta, \mathcal{E})\right], 0\right\}
\end{aligned}
$$

where $\lambda$ and $r_{X}$ are considered as functions of $\mathcal{E}$ and $\theta$ due to Eqs. (33) and (35). A more exact expression for $\Gamma(\theta, \mathcal{E}) \Gamma$ which takes into account the finite Larmor radius $\Gamma$ is given in Appendix A.

\section{COMPARISON OF THEORETICAL RESULTS WITH EXPERIMENTAL DATA}

According to Fig. 35 sawtooth oscillations almost do not affect the alpha flux at the $45^{\circ}$ detector. This experimental fact indicates that two dominant mechanisms which were considered in the subsection IV A Tnamely the crash-induced prompt loss and the stochastic diffusion lead to irradiation of different parts of the wall.

In order to verify this hypothesis $\Gamma$ the role of the crash-induced stochastic diffusion has been evaluated. With this purpose $\Gamma$ using the Hamiltonian guiding-center code ORBITI ${ }^{8,19}$ we have calculated the fraction of lost particles for the following profile shape of the alpha source $G$ : $G=\left(1-r^{2} / a^{2}\right)^{2} \eta(0.4-r / a) \Gamma$ where $\eta$ is the Heavyside function. When choosing this function we took into account that a sawtooth crash expel a particle into the stochastic loss region mainly at $r<r_{m i x}$. The safety factor $q(r)$ was taken in the form

$$
q=q_{0}+q_{1} r / a+q_{2}(r / a)^{2}+q_{3}(r / a)^{3}
$$

with $q_{0}=0.7 \Gamma q_{1}=0.55 \Gamma q_{2}=-0.84 \Gamma q_{3}=4.35 \Gamma$ which was obtained by interpolation of experimental measurements for the shot \#87530. The results of the calculations for particles with $\mathcal{E}=3.5 \mathrm{MeV}$ are shown in Fig. 12. Two main conclusions can be drawn from this figure. First $\Gamma$ the particles are lost within the time which is small in comparison with their slowing-

down time (the energy spectrum of the escaping alphas is strongly peaked at the birth 
energy). Second $\Gamma$ the bulk of the escaping $3.5-\mathrm{MeV}$ particles is constituted by moderately trapped alphas with $\lambda=1$.1. In order to find the dependence of the sawtooth-induced ripple loss on the particle energy the calculations have been carried out also for $\mathcal{E}=1.6 \mathrm{MeV}$. It has been found that the lost fraction of these particles is less than that of the $3.5-\mathrm{MeV}$ particles almost by a factor of 10 . Thus 5 the main fraction of the lost alphas consists of high-energy trapped particles with $\mathcal{E} \sim 3 \mathrm{MeV}$ and $\lambda \sim 1$.1. Using this fact $\Gamma$ we conclude from Fig. 11 that the crash-induced stochastic diffusion can lead to noticeable alpha flux only at $20^{\circ}$ detector.

Taking this into account $\Gamma$ we will analyze the poloidal distribution of the alpha flux to the wall at $\theta \geq 45^{\circ}$ Tusing Eq. (38) $\Gamma$ which describes the crash-induced prompt loss of alphas. The results of calculations for the shots \#87529 and \#87530 are presented in Figs. 13-15. These discharges were characterized by almost identical plasma parameters. Two sawteeth in the discharge \#87529 and three sawteeth in the discharge \#87530 were accompanied by loss of alpha particles. In our calculations $\Gamma$ we take the $q$ profile given by Eq. (39) and $\beta=0.3 \%$. From Eq. (13) $\Gamma$ we obtain that $\Delta^{\prime}=1.67 A^{-1}$. Two crash models were used: first $\Gamma$ the Kadomtsev model [see Eq. (32) for $\left.V_{0}\left(r_{X}\right)\right]$ and $\Gamma$ second $\Gamma$ the model with $V_{0}\left(r_{X}\right)=$ const. The latter takes place when the plasma is strongly mixed during the crash. Therefore $\Gamma$ we refer to this model as to the "strong-mixing model".

The figures 13 and 14 show the poloidal dependence of the flux of the monoenergetic alpha particles to the wall for various energies and the two crash models. In order to study the sensitivity of the results to the Shafranov shift $\Gamma$ the calculations were carried out for various values of $\Delta^{\prime}$. It follows from these figures that all the distributions are decreasing (except for the region $|\theta|>100^{\circ} \Gamma$ where detectors are absent). The decrease is the strongest for the particles with the smallest energy. The calculated flux is very small at the $45^{\circ}$ detector $\Gamma$ which agrees with experimental data. We find that the poloidal dependence of $\Gamma_{\mathcal{E}}$ is rather sensitive to the crash modelГespeciallyГfor particles of low energy in the region of $\theta \sim 90^{\circ}$.

The figure 15 shows the energy distribution of alpha particles at the $90^{\circ}$ and $60^{\circ}$ detectors 
for two crash models. We observe that the Kadomtsev model yields non-monotonic energy dependence at the $90^{\circ}$ detector with the maximum at $\mathcal{E} / \mathcal{E}_{\alpha} \approx 0.7$ but monotonic dependence at the $60^{\circ}$ detector $\Gamma$ with the alpha flux $\Gamma_{\mathcal{E}}$ being almost constant at the interval $0.6 \leq \mathcal{E} / \mathcal{E}_{\alpha} \leq$ 1. Both models predict vanishing flux for $\mathcal{E} / \mathcal{E}_{\alpha}=0.22$ and 0.45 at the $90^{\circ}$ and $60^{\circ}$ detectors $\Gamma$ respectively.

FinallyГFig. 16 presents results of calculations of the poloidal distribution of the integral alpha flux density for the Kadomtsev model. The calculations were carried out for $\Delta^{\prime}=1.67 A^{-1} \Gamma \Delta^{\prime}=1.8 A^{-1} \Gamma$ and $\Delta^{\prime}=2 A^{-1}$. In addition $\Gamma$ the figure shows the results of the experimental measurements of the alpha flux produced by three crashes in the shot \#87530 at the $90^{\circ} \Gamma 60^{\circ}$ and $45^{\circ}$ detectors. The experimental data are normalized by the data obtained from the $90^{\circ}$ detector. The calculations were carried out also for the strong-mixing model but it turned out that the poloidal distribution of the integral alpha flux Tunlike the monoenergetic flux Tis practically insensitive to the crash model. The calculations predict the best agreement with the experimental measurements at all three detectors for $\Delta^{\prime}=1.8 A^{-1} \Gamma$ which is close to $\Delta^{\prime}=1.67 A^{-1}$ following from Eq. (13). The obtained distributions are strongly sensitive to $\Delta^{\prime}$. Taking into account uncertainties in both the model we use and the experimental data we can conclude that the calculations are in reasonable agreement with the experiment.

Thus the experimentally observed poloidal distributions of the alpha flux presented in Fig. 3 can be explained in terms of the crash-induced prompt loss and stochastic diffusion.

Now we consider the time dependence of the alpha flux presented in Fig. 2. This figure demonstrates correlation between the temporal variation of the alpha signal at the wall and the electron temperature $\left(T_{e}\right)$ at some radii during the sawtooth crash. We observe that the alpha signal at the $60^{\circ}$ detector correlates with the change of $T_{e}$ at $R \leq 2.86 \mathrm{~m}$ Whereas the alpha signal at the $90^{\circ}$ detector has two maxima and correlates with the variation of $T_{e}$ at $R=2.98 \mathrm{~m}$.

In order to explain this phenomenon we use again the model of the crash-induced prompt 
loss. Using Eqs. (33)-(36) $\Gamma(\mathrm{A} 1) \Gamma(\mathrm{A} 2)$ (see Appendix A for details) $\Gamma$ we find for the shots \#87529 and \#87530 that the $3.5-\mathrm{MeV}$ alpha particles reaching the $90^{\circ}$ and $60^{\circ}$ detectors are characterized by $\lambda=0.81 \Gamma r_{X}=46 \mathrm{~cm}$ and $\lambda=0.84 \Gamma r_{X}=39 \mathrm{~cm} \Gamma$ respectively. This means that the point of the orbit transformation of the particles coming to the $60^{\circ}$ detector is located closer to the plasma center than that of the particles coming to the $90^{\circ}$ detector $\left(R_{t}=\lambda R_{0}\right)$. Unfortunately $\Gamma$ experimental data about the evolution of $T_{e}$ at $R<R_{0}$ are

not available. Therefore $\Gamma$ we have to make some assumptions concerning the structure of the perturbation in order to explain correlation of the alpha signal with the change of $T_{e}$ at $R>R_{0}$. We assume that the helical symmetry is approximately conserved during the crash. In addition $\Gamma$ we take into account that the particle orbit width is relatively small before the transformation and their bounce period is much less than the crash duration. Then we conclude that the moment of the enhanced alpha signal at the wall approximately coincides with the moment of the sharp change of $T_{e}$ at $R_{1}(\theta)=R_{0}+r_{X}(\theta)$. Taking $R_{0}=252 \mathrm{~cm} \Gamma$ we obtain that $R_{1}\left(90^{\circ}\right)=298 \mathrm{~cm} \Gamma$ which equals to the radius where the experimentally observed perturbation of the electron temperature correlates with the alpha signal at the $90^{\circ}$ detector. On the other hand $\Gamma R_{1}\left(60^{\circ}\right)=291 \mathrm{~cm} \Gamma$ and the electron temperature spike at the closest available observation point $R=286 \mathrm{~cm}$ correlates with the observed alpha flux burst at $\theta=60^{\circ}$.

\section{SUMMARY AND CONCLUSIONS}

We have investigated both experimentally and theoretically the effect of sawtooth oscillations on confinement of fast ions. The results can be summarized as follows.

Fusion produced alpha particles escaping because of crashes of sawtooth oscillations in TFTR DT shots have been analyzed by detectors located at $90^{\circ} \Gamma 60^{\circ} \Gamma 45^{\circ} \Gamma 20^{\circ}$ below the midplane of the torus. It has been found that only a small fraction of alphas is expelled from the plasma and $\Gamma$ thus $\Gamma$ the main effect of sawteeth on alpha particles in TFTR is their 
redistribution within the plasma volume. The measured poloidal distributions of the alpha flux are very inhomogeneous $\Gamma$ being strongly peaked at $\theta=-90^{\circ}$ (i.e. $\Gamma$ near the bottom of the vessel) and $\theta=-20^{\circ}$ Tand almost not noticeable at $\theta=-45^{\circ}$.

The analysis has been carried out $\Gamma$ which shows that the main physical mechanism resulting in the alpha flux to the $20^{\circ}$ detector is the crash-induced stochastic (collisionless) diffusion. The stochastic diffusion leads to escape of moderately trapped alpha particles $(\lambda \sim 1)$ after the sawtooth crash displaces them to the region of sufficiently large ripple of the toroidal magnetic field. The diffusion is so strong that the alphas escape from the plasma for the time small in comparison with the slowing-down time. However $\Gamma$ it yields almost no flux of alpha particles to the wall area $|\theta| \geq 45^{\circ} \Gamma$ where three detectors were located. Therefore $\Gamma$ in our study of the poloidal wall load at $|\theta| \geq 45^{\circ} \Gamma$ the magnetic ripple was neglected.

In this approximation $\Gamma$ the following analysis has been fulfilled.

A numerical simulation of the motion of single alpha particles in a TFTR DT plasma has been carried out. The simulation was based on the modeling of sawtooth crash according to Ref. ${ }^{6}$ and a new simple model of the magnetic field in the vacuum gap region suggested in the present work. It revealed the mechanism of expulsion of fast ions to the wall and showed which particles can be expelled. In particularTit has been found that only marginally circulating particles located well inside the sawtooth mixing region before the crash can be lost. Such particles are displaced by the crash outwards $\Gamma$ which leads to transformation of their orbits to marginally trapped ones reaching the wall. Furthermore $\mathrm{Tit}$ has been shown that only particles with sufficiently high energy $\Gamma \mathcal{E} \geq \mathcal{E}_{\min }$ can be lost $\Gamma$ and that the lost particles strike the wall at $\theta_{\min }<|\theta|<\theta_{\max }$ (either below or above the midplane of the torus $\Gamma$ depending on the direction of the toroidal magnetic field). The orbits of all lost particles intersect the wall except for the particles with $\mathcal{E}=\mathcal{E}_{\min }$ and particles reaching the wall at $\theta=\theta_{\tau}(\mathcal{E}) \Gamma$ whose orbits are tangent to the wall $\left[\theta_{\min }=\min \theta_{\tau}(\mathcal{E})\right]$.

The factors leading to existence of $\theta_{\min }$ and $\theta_{\max }$ are different. $\theta_{\max }$ is associated with 
the fact that trapped particles can strike the wall only at a point located at $R>R_{t}$. On the other hand $\Gamma$ the crash-induced flux of alpha particles to the wall is vanishing for $\theta<\theta_{\min }$ because of the wall shadowing produced by the vacuum gap. Note that the shadowing effect exists even without the gap. It takes place in all cases when a particle orbit intersects the wall in more than one point: such a particle is lost at the first wall point which is met during the orbital motion and $\Gamma$ therefore $\Gamma$ all other points where the orbit intersects the wall are in the shadow. In this sense all wall region above/below the midplane of the torus is shadowed for prompt losses. The vacuum gap produces an addition shadow Treducing the region where particles can reach the wall. This effect becomes important $\Gamma$ i.e. $\Gamma \theta_{\min }$ is not close to zero $\Gamma$ provided that the vacuum gap is not too small and the particle energy is not too high follows from a condition obtained in the present work. This condition is satisfied for alpha particles in TFTR.

Note that the obtained condition is general and can be applied for a particle lost due to any physical mechanism (except for the case when the particle orbit is strongly perturbed during the last bounce before striking the wall). It follows from this condition that the shadow for particles with given $\mathcal{E}$ increases with $\lambda$. For this reason $\Gamma$ the shadow is minimum for the particles escaping to the ripple diffusion (in fact $\Gamma$ the gap-induced shadow is absent for particles with $\mathcal{E} \sim 3 \mathrm{MeV}$ ) and maximum for the alpha loss associated with the orbit transformation. Just this circumstance explains why the alpha flux to the $20^{\circ}$ detector and the flux to the $60^{\circ}$ and $90^{\circ}$ detectors are associated with different physical mechanisms.

A general conclusion which can be drawn from the described results of numerical simulations is that the crash-induced prompt loss of alpha particles is responsible for the alpha wall load far from the midplane of the torus. To describe the poloidal distribution of the alpha flux associated with this mechanism $\Gamma$ an approach is suggested $\Gamma$ which employs the fact that the process of the particle escape to the wall includes two stages observed in numerical simulations (i.e. Tthe motion before and after the orbit transformation). General expressions for the alpha flux to the wall are obtained which are applied to crashes satisfying the inequality 
$\tau_{c r} \gg \tau_{b}$

Based on this approach $\Gamma$ the alpha wall load poloidal distribution $\Gamma \Gamma(\theta) \Gamma$ has been calculated for the TFTR DT shot. The obtained results are in qualitative agreement with the experimental data. In addition $\Gamma$ the monoenergetic poloidal distribution of the alpha flux $\Gamma$ $\Gamma(\theta, \mathcal{E}) \Gamma$ has been obtained. The flux has been calculated for two crash models: first $\Gamma$ the Kadomtsev model and $\Gamma$ second $\Gamma$ for $V\left(r_{X}\right)=$ const (which corresponds to very strong plasma mixing). It has been found that the monoenergetic distribution is rather sensitive to the crash model $\Gamma$ whereas the poloidal dependence of the integral alpha flux is not.

Note that the physical picture of the prompt escape of fast ions caused by core localized MHD perturbations of different kinds seems to be similar to the considered one Cinvolving two stages. At the first stage $\Gamma$ the particle motion is strongly affected by the interaction with a perturbation. This stage is different for the MHD perturbations of different kinds. However $\Gamma$ the second stage $\Gamma$ i.e. Tthe motion along orbits of marginally trapped particles in the loss-cone regionTis similar. This may explain why the poloidal distributions of alpha flux to the wall at $|\theta| \geq 45^{\circ}$ measured experimentally in TFTR are rather similar for the losses induced by sawtooth oscillations $\Gamma$ Mirnov oscillations and minor disruptions. ${ }^{20}$ The differences at the first stage may explain very different wall load magnitude due to the mentioned processes.

In spite of the fact that only a narrow group of particles is expelled to the wall $\Gamma$ the sawtooth-induced transport of fast ions may be of practical importance because of the following. First Tit flattens the energy profile deposition of fast ions. This effect takes place not only inside the sawtooth mixing region but also for $r=r_{m i x}+\delta r_{b} \Gamma$ where $\delta r_{b}$ is the characteristic orbit width of fast ions. This is illustrated by Figs. 5-8 5 which demonstrate that a considerable part of the orbit of a particle located before a crash in the sawtooth mixing region can lie after the crash at $r>r_{m i x}$. Second $\Gamma$ the alpha flux the wall $\Gamma$ especially the monoenergetic flux $\Gamma(\theta, \mathcal{E})$ is sensitive to the crash type; therefore $\Gamma$ it can be used for studying the nature of the sawtooth crash. 


\section{ACKNOWLEDGMENTS}

The research described in this publication was made possible in part by the Research Contract No. 8924 of the International Atomic Energy Agency and the Award No. UP2-290 of the Government of Ukraine and the U.S. Civilian Research \& Development Foundation for the Independent States of the Former Soviet Union (CRDF). Any opinions $\Gamma$ findings $\Gamma$ conclusions or recommendations expressed in this material are those of the authors and do not necessarily reflect those of the Government of Ukraine and CRDF.

Two of the authors (Y.K. and Y.Y.) would like to acknowledge the hospitality of the Princeton Plasma Physics Laboratory.

\section{APPENDIX A: THE EFFECT OF THE LARMOR RADIUS ON THE PARTICLE FLUX TO THE WALL.}

The cyclotron frequency of the alpha particles in TFTR is Tat least $\Gamma$ two orders of magnitude higher than their bounce frequency. For this reason $\Gamma$ we will assume that a particle strikes the wall at the moment when its Larmor circle touches the wall is at the distance of the Larmor radius $\Gamma \rho_{\perp}=\rho(\lambda h)^{1 / 2} \Gamma$ from the wall. Hence $\Gamma$ we obtain the corresponding value of $h$ at the guiding center:

$$
h_{w}=1+\frac{\Delta_{w}}{R_{0}}+\frac{r_{w}-\rho_{\perp}(\lambda, \theta)}{R_{0}} \cos (\theta) .
$$

Note that here and below the subscript " $w$ " refers to the particle guiding center at the moment of the particle escape. The flux surface radius $\Gamma r \Gamma a t$ this point can be obtained from the equation

$$
r_{w}^{2}=\left(a_{c}-\rho_{\perp}\right)^{2}+\left(\Delta_{w}+\Delta_{c}\right)^{2}+2\left(a_{c}-\rho_{\perp}\right)\left(\Delta_{w}+\Delta_{c}\right) \cos (\theta)
$$

[cf. Eq. (15)]. Substituting Eqs. (36) and (A1) into the definition of JTdifferentiating over $\theta$ Tand taking into account Eq. (A2) $\Gamma$ which relates $r_{w}$ to $\theta$ Гwe obtain 


$$
\frac{\partial J}{\partial \theta}(\theta, \mathcal{E}, \lambda)=-M a_{c} v \sin (\theta)\left\{\frac{1+\chi_{w}^{2}}{2 \chi_{w}}\left[1-\frac{\rho_{\perp}}{a_{c}}-\frac{\rho \lambda^{1 / 2}}{2 R_{0} h_{w}^{1 / 2}} \cos (\theta)\right]-\mathcal{G}\right\},
$$

where $\mathcal{G}$ is now redefined as follows:

$$
\begin{gathered}
\mathcal{G}(\theta, \mathcal{E}, \lambda)=\left.\frac{1}{B_{0} Q\left(r_{w}, \theta\right)}\left[\left(1-\frac{\rho_{\perp}}{a_{c}}\right) \frac{\Delta_{w}+\Delta_{c}}{\rho}+Y \frac{a_{c} \lambda^{1 / 2}}{2 R_{0} h_{w}^{1 / 2}}\right] \frac{d \Psi_{p}}{d r}\right|_{r=r_{w}(\theta)}, \\
Q(r, \theta)=r-\Delta^{\prime}\left[\Delta(r)+\Delta_{c}+a_{c} \cos (\theta)\right], \\
Y(\theta, \mathcal{E}, \lambda)=1-\frac{\rho_{\perp}}{a_{c}}+\frac{\Delta_{w}+\Delta_{c}}{a_{c}} \cos (\theta) .
\end{gathered}
$$

Solving the equation

$$
\frac{\partial J}{\partial \theta}\left(\theta_{\tau}, \mathcal{E}, \lambda\right)=0
$$

for $\theta_{\tau}$ at given $\mathcal{E}$ and $\lambda$ Гone can calculate $\theta_{\tau}(\mathcal{E}, \lambda)$.

In order to find the Larmor corrections to Eq. (38) Гwe substitute the value at the Larmor center [Eq. (A1)] for $h_{w}$ in Eqs. (33) $\Gamma(36)$. Differentiating Eqs. (33)-(36) $Г(\mathrm{~A} 2)$ at fixed energy and excluding the differentials of $r_{X} \Gamma v_{\| w} \Gamma v_{\| X} \Gamma$ and $r_{w} \Gamma$ we can find $\partial \lambda / \partial \theta$. Then $\Gamma$ using Eqs. $(27) \Gamma(30) \Gamma$ we obtain:

$$
\begin{aligned}
\Gamma(\theta, \mathcal{E}) & =-\sin (\theta) \frac{f v^{2} q_{X} \rho V\left(r_{X}\right) \Xi}{2 r_{X} R_{0}^{2}(1-\lambda)^{1 / 2} h_{w}} \\
& \times \max \left\{\left[\frac{1+\chi_{w}^{2}}{2 \chi_{w}}\left(1-\frac{\rho_{\perp}}{a_{c}}-\frac{\rho \lambda^{1 / 2}}{2 R_{0} h_{w}^{1 / 2}} \cos (\theta)\right)-\mathcal{G}\right], 0\right\} \\
& \times\left[1+\frac{q_{X} \rho \Xi}{2 r_{X} \chi_{w}}+\frac{R_{0} \rho^{2} q_{X}^{2}}{2 r_{X}^{3}}\left(h_{X} S_{X}-1\right)+\frac{q_{X} \rho^{2} h_{w}^{1 / 2} \Xi}{2 R_{0} r_{X} \chi_{w} \lambda^{1 / 2}} \frac{1+\chi_{w}^{2}}{2 \chi_{w}} \cos (\theta)\right. \\
& \left.+\left.\frac{q_{X} \rho a_{c} h_{w}^{1 / 2} \Xi Y}{2 R_{0} r_{x} Q\left(r_{w}, \theta\right) B_{0} \lambda^{1 / 2}} \frac{d \Psi_{p}}{d r}\right|_{r=r_{w}(\theta)}\right]^{-1},
\end{aligned}
$$

where $S=(r / q) d q / d r$ is the magnetic shear $\Gamma$

$$
\Xi=1-\frac{\rho^{2} q_{X}^{2}}{4 r_{X}^{2}}+\frac{h_{X} R_{0} \rho^{2} q_{X}^{2}}{2 r_{X}^{3}}\left(S_{X}-1\right) .
$$

The equation (A8) was used in Sec. $\mathrm{V}$ to calculate the alpha flux to the wall. The procedure of the calculations was the following. Given $\theta \Gamma \mathcal{E} \Gamma$ and $r_{X} \Gamma$ one can calculate 
$\lambda \Gamma v_{\| X} \Gamma v_{\| w} \Gamma h_{w} \Gamma$ and $r_{w}$ from Eqs. (34)-(36) $(\mathrm{A} 1) \Gamma$ and (A2). Then Eq. (33) becomes an equation relating $\theta \Gamma \mathcal{E} \Gamma$ and $r_{X}$. Solving it for $r_{X}$ at given $\theta$ and $\mathcal{E}$ and substituting the obtained value into the mentioned equations Tone can find all quantities that are required to calculate the right-hand side of Eq. (A8). 


\section{REFERENCES}

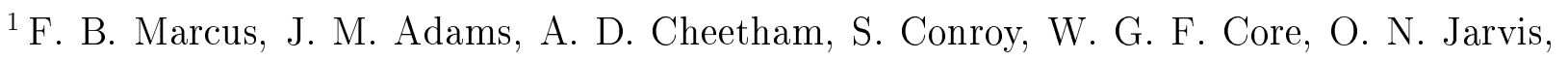
M. J. Loughlin Г G. SadlerГ P. Smeulders $\Gamma$ P. van Belle $\Gamma$ and N. Watkins $\Gamma$ Plasma Phys. Controlled Fusion 33Г277 (1991).

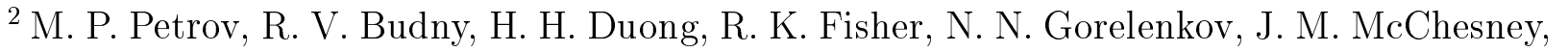
D. K. MansfieldГS. S. MedleyГP. B. ParksГM. H. RediГand A. L. RoquemoreГNucl. Fusion 35Г1437 (1995).

${ }^{3}$ O. N. JarvisГJ. M. AdamsГP. J. A. HowarthГF. B. MarcusГE. RighiГG. J. SadlerГD. F. H. StartГP. van BelleГC. D. WarrickГand N. WatkinsГNucl. Fusion 36Г1513 (1996).

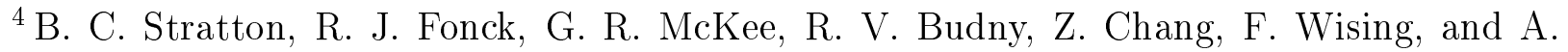
Ödblom N Nucl. Fusion 36Г1586 (1996).

${ }^{5}$ Ya. I. Kolesnichenko and Yu. V. YakovenkoГNucl. Fusion $32 \Gamma 449$ (1992).

${ }^{6}$ Ya. I. Kolesnichenko and Yu. V. YakovenkoГReport at the IV International Atomic Energy Agency Technical Committee Meeting on Alpha Particles in Fusion Research (Princeton $\Gamma$ April 1995); Nucl. Fusion 36Г159 (1996).

${ }^{7}$ Yi Zhao and R. B. WhiteГPhys. Plasmas 4Г1103 (1997).

${ }^{8}$ D. J. Grove and D. M. MeadeГNucl. Fusion 25Г1167 (1985).

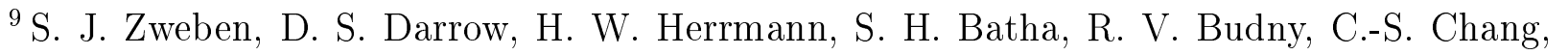

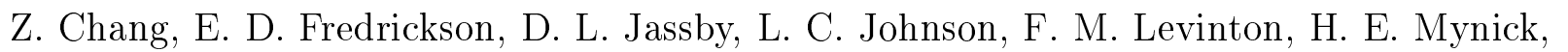

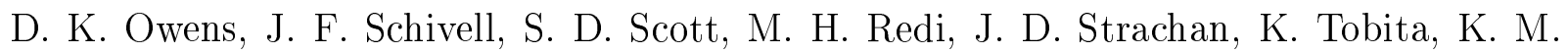
Young NNucl. Fusion 35Г893 (1995).

${ }^{10}$ S. J. ZwebenГD. S. DarrowГS. H. BathaГR. V. BudnyГM. DiessoГH. W. HerrmannГJ. GiarrussoГM. H. RediГH. TakahashiГS. von GoelerГR. B. WhiteГR. M. WielandГNucl. Fusion 38Г739 (1998). 


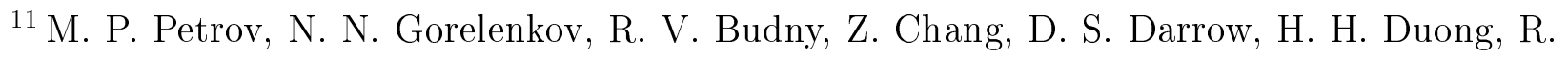
K. FisherГR. J. FonckГH. W. HerrmannГG. R. McKeeГS. S. MedleyГA. ÖdblomГA. L.

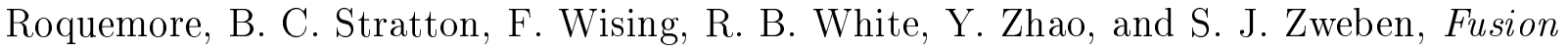
Energy 1996, Proceedings of the 16th International Atomic Energy Agency Conference, Montreal, 1996 (International Atomic Energy AgencyГViennaГ1997)ГVol. 1Гp. 261.

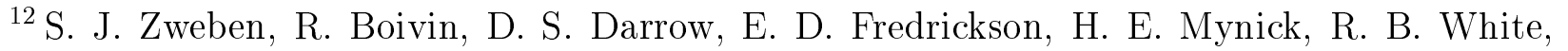

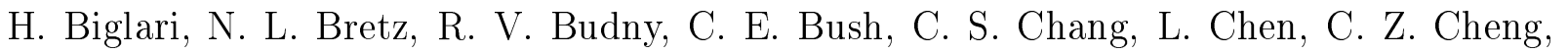

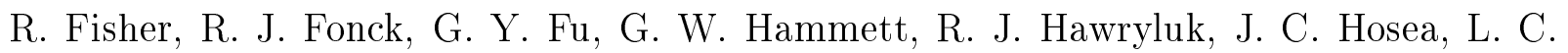
JohnsonГJ. S. MachusakГD. K. MansfieldГJ. McChesneyГK. M. McGuireГG. McKeeГS. S. MedleyГM. MurakamiГR. NazikianГD. K. OwensГH. K. ParkГJ. ParkГC. K. PhillipsГJ.

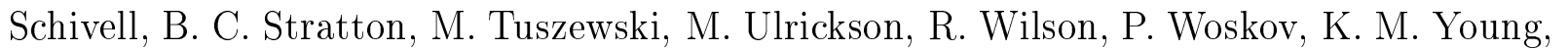
Plasma Physics and Controlled Nuclear Fusion Research 1992, Proceedings of the 16th International Atomic Energy Agency Conference, Würzburg, 1992 (International Atomic

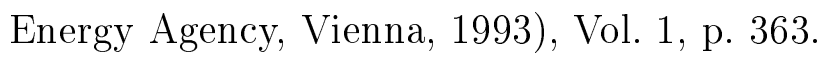

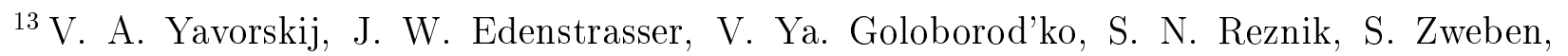
Proceedings of the Fifth International Atomic Energy Agency Technical Committee Meeting on Alpha Particles in Fusion Research, Abingdon, 1997 (International Atomic Energy AgencyГViennaГ1997) 29.

${ }^{14}$ L. E. Zakharov and V. D. ShafranovTin Problems of Plasma Theory (Consultants Bureau New YorkГ1986)ГVol. 11Гp. 153.

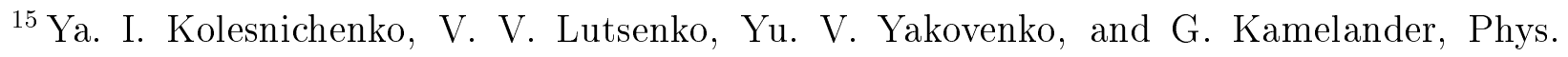
Plasmas 4Г2544 (1997).

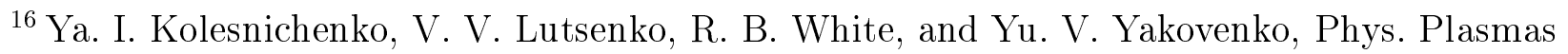
$5 \Gamma 2963$ (1998).

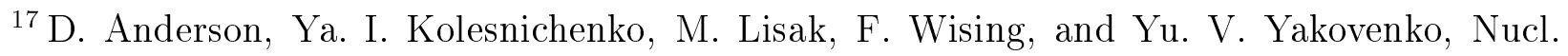


Fusion 34Г217 (1994).

${ }^{18}$ R. B. White and M. S. ChanceГPhys. Fluids 27Г2455 (1984).

${ }^{19}$ R. B. WhiteГPhys. Fluids B 2Г845 (1990).

${ }^{20}$ S. J. ZwebenГ Z. ChangГ D. S. DarrowГ E. D. FredricksonГ W. ParkГ E. RuskovГ J. D. StrachanГH. TakahashiГand R. B. WhiteГReport 1WepO1 5 at the 39th Annual Meeting of the Division of Plasma Physics of the American Physical Society (PittsburghГ1997); Bull. Am. Phys. Soc. 42Г1971 (1997). 


\section{FIGURES}

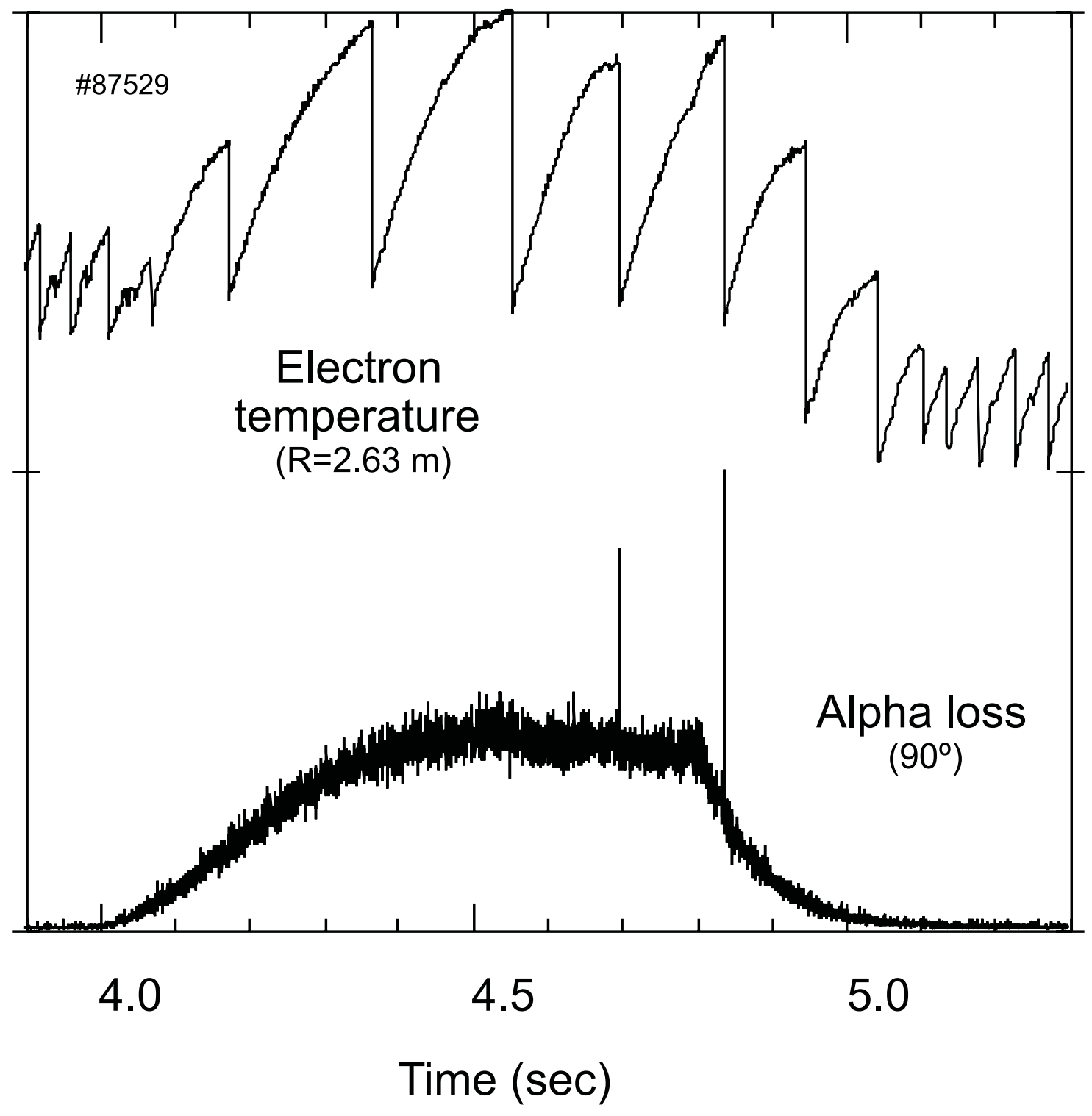

FIG. 1. 


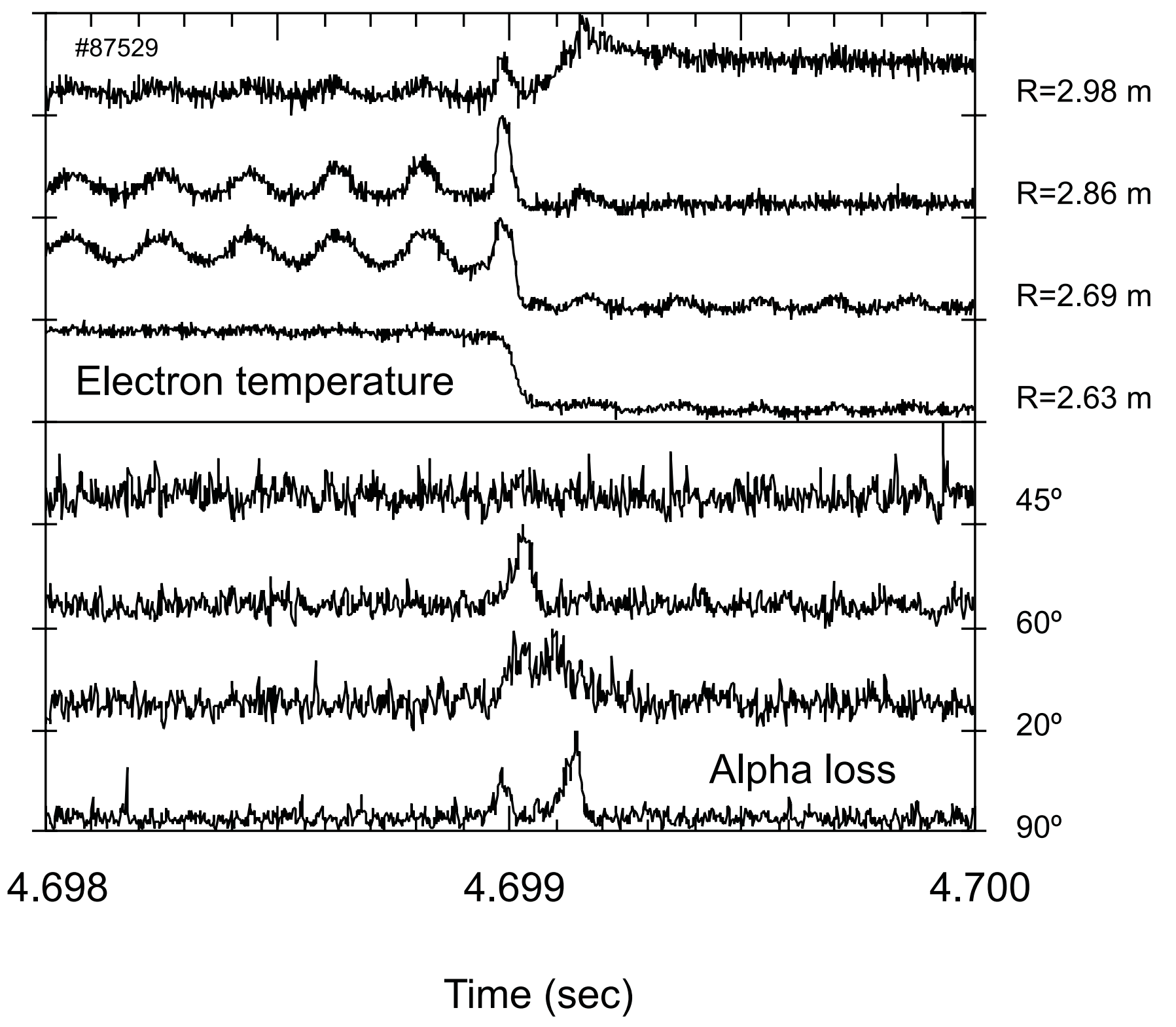

FIG. 2. 

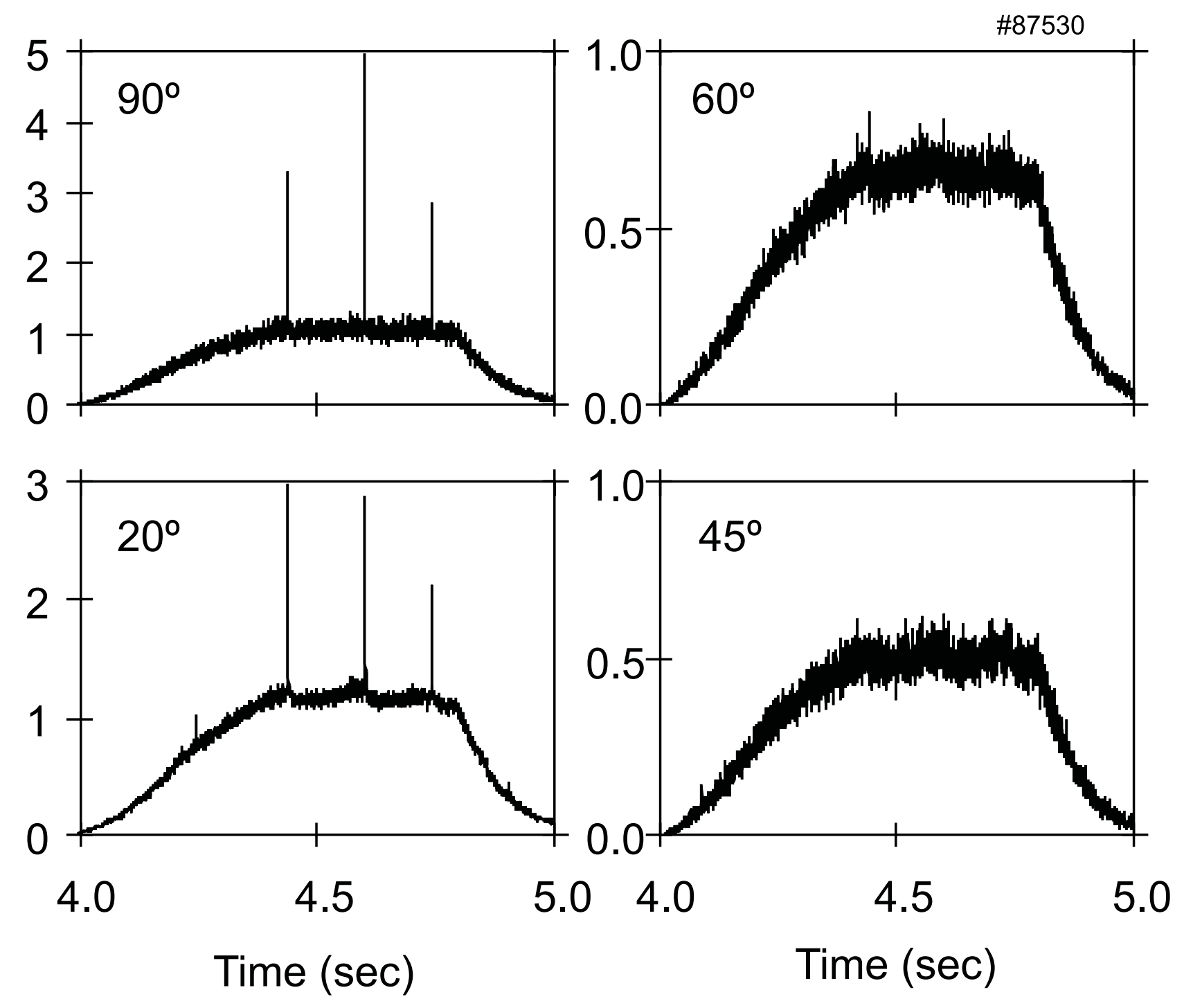

FIG. 3a. 

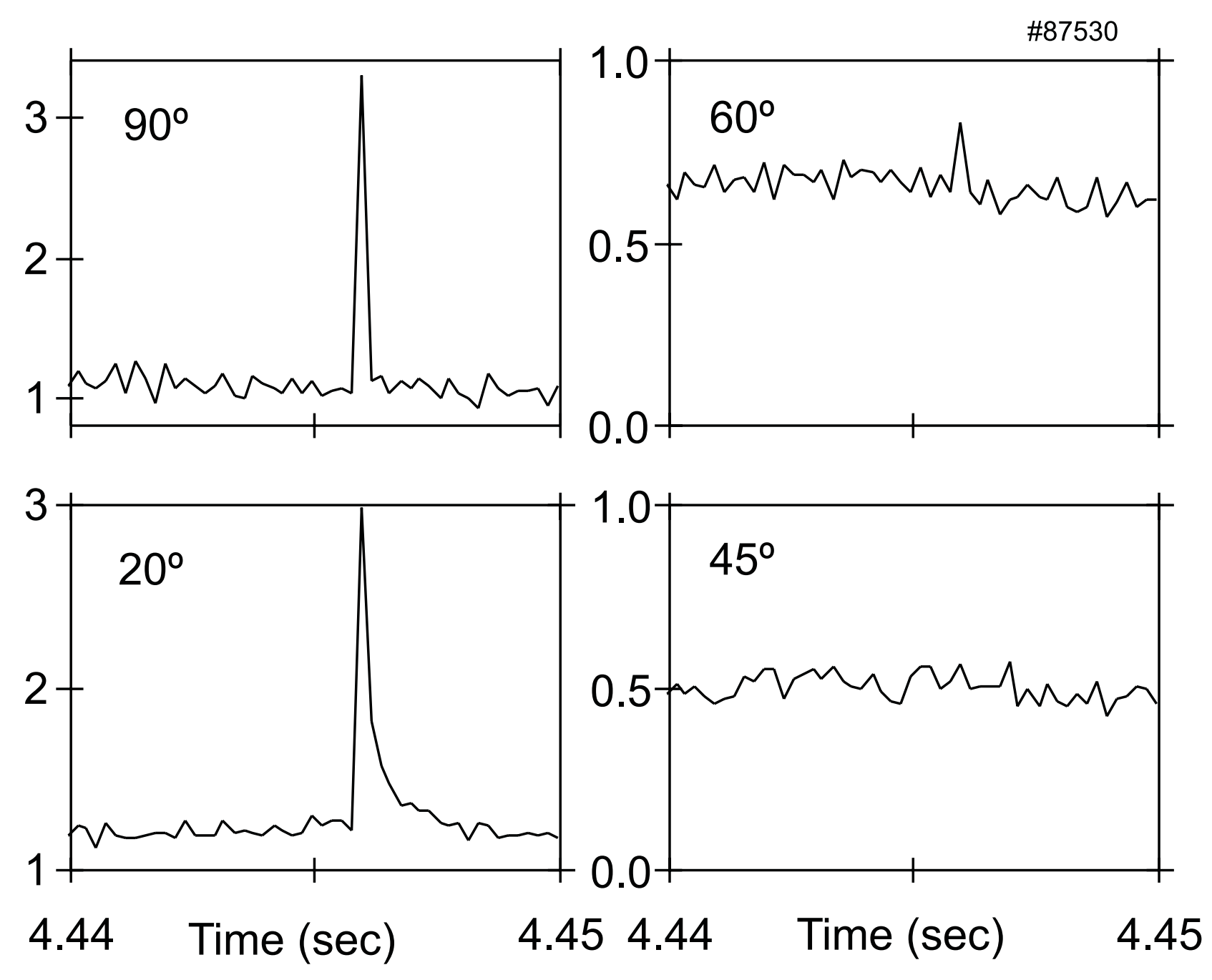

FIG. 3b. 


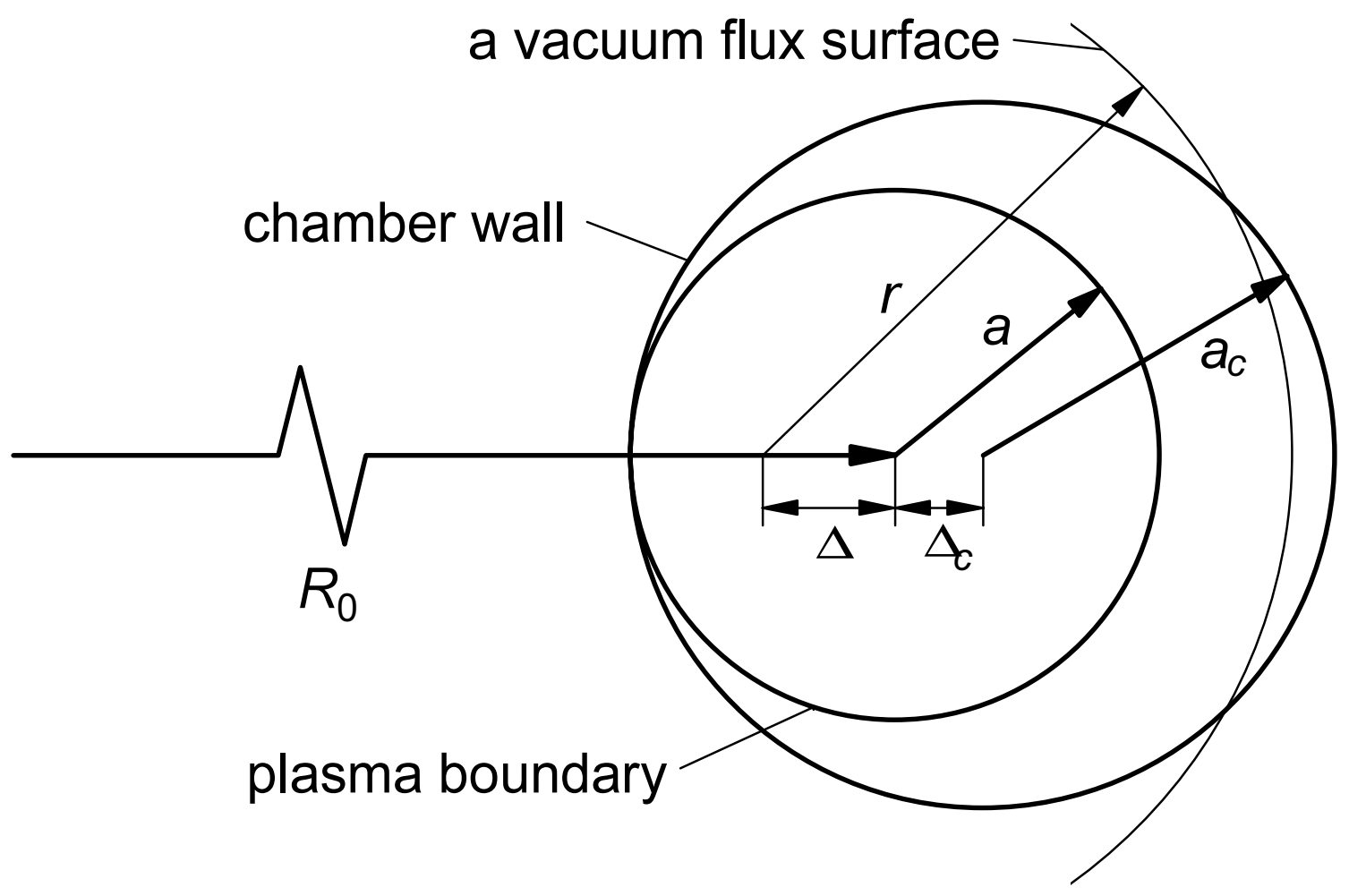

FIG. 4. 


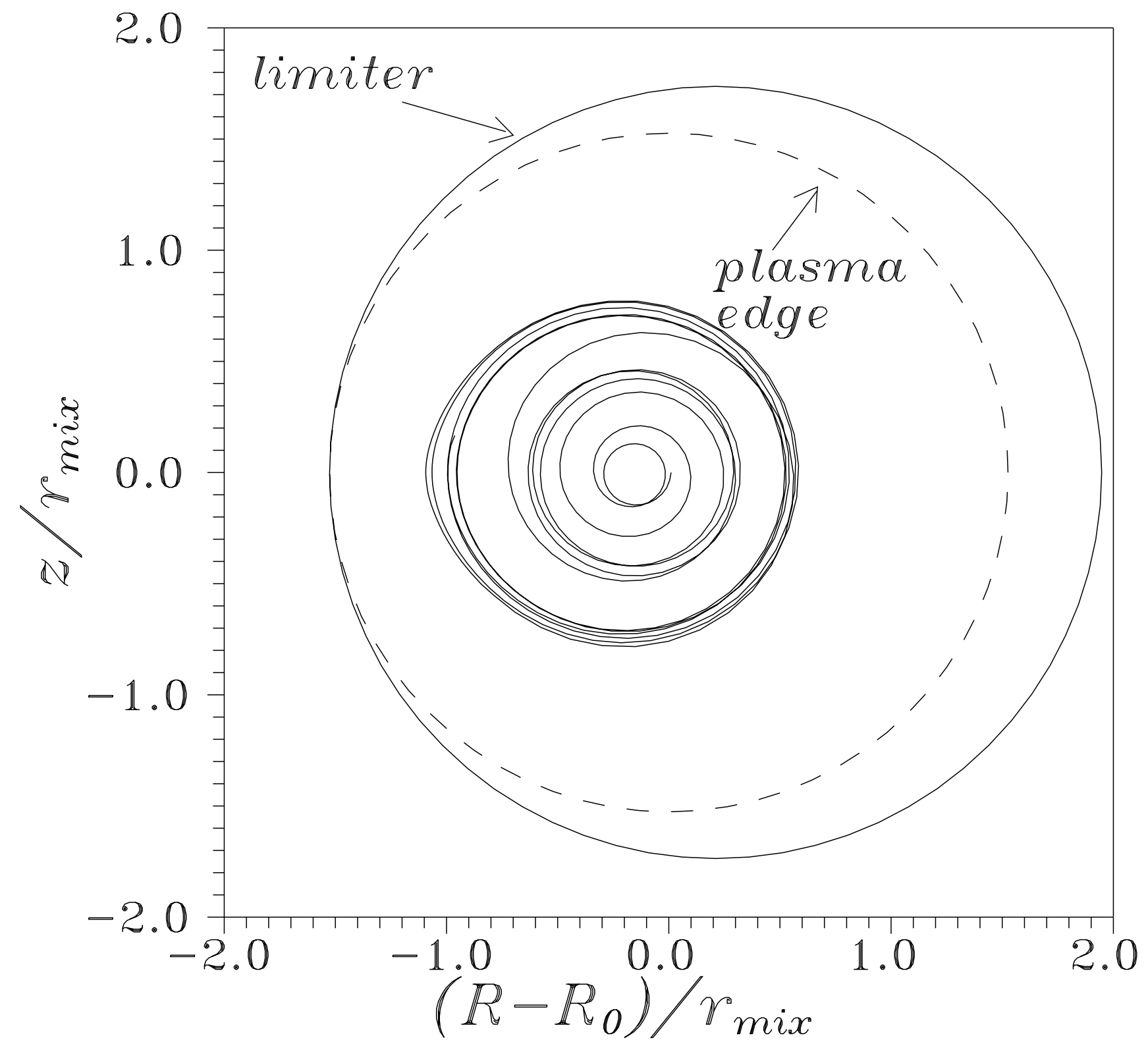

FIG. 5a. 


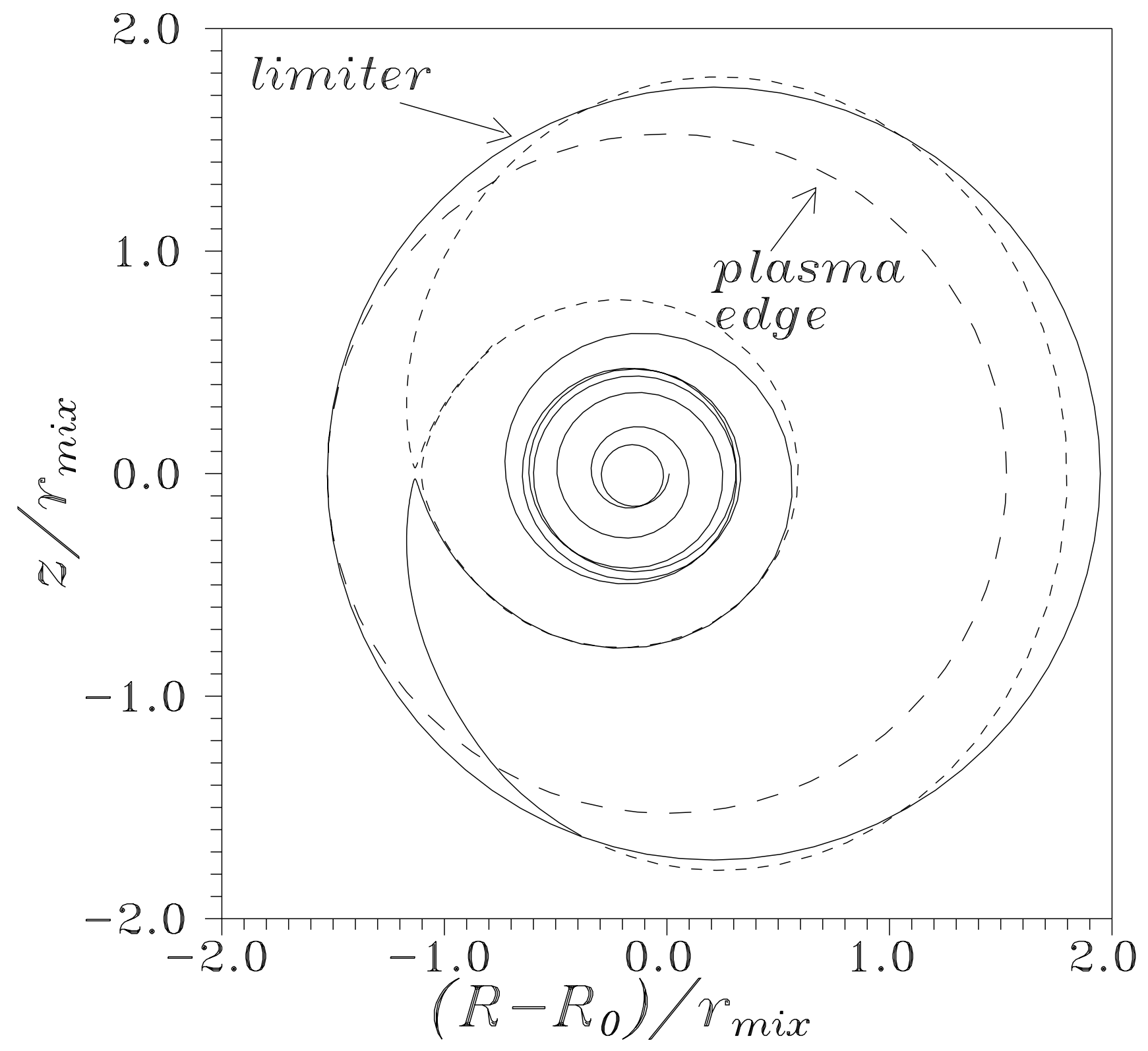

FIG. 5b. 


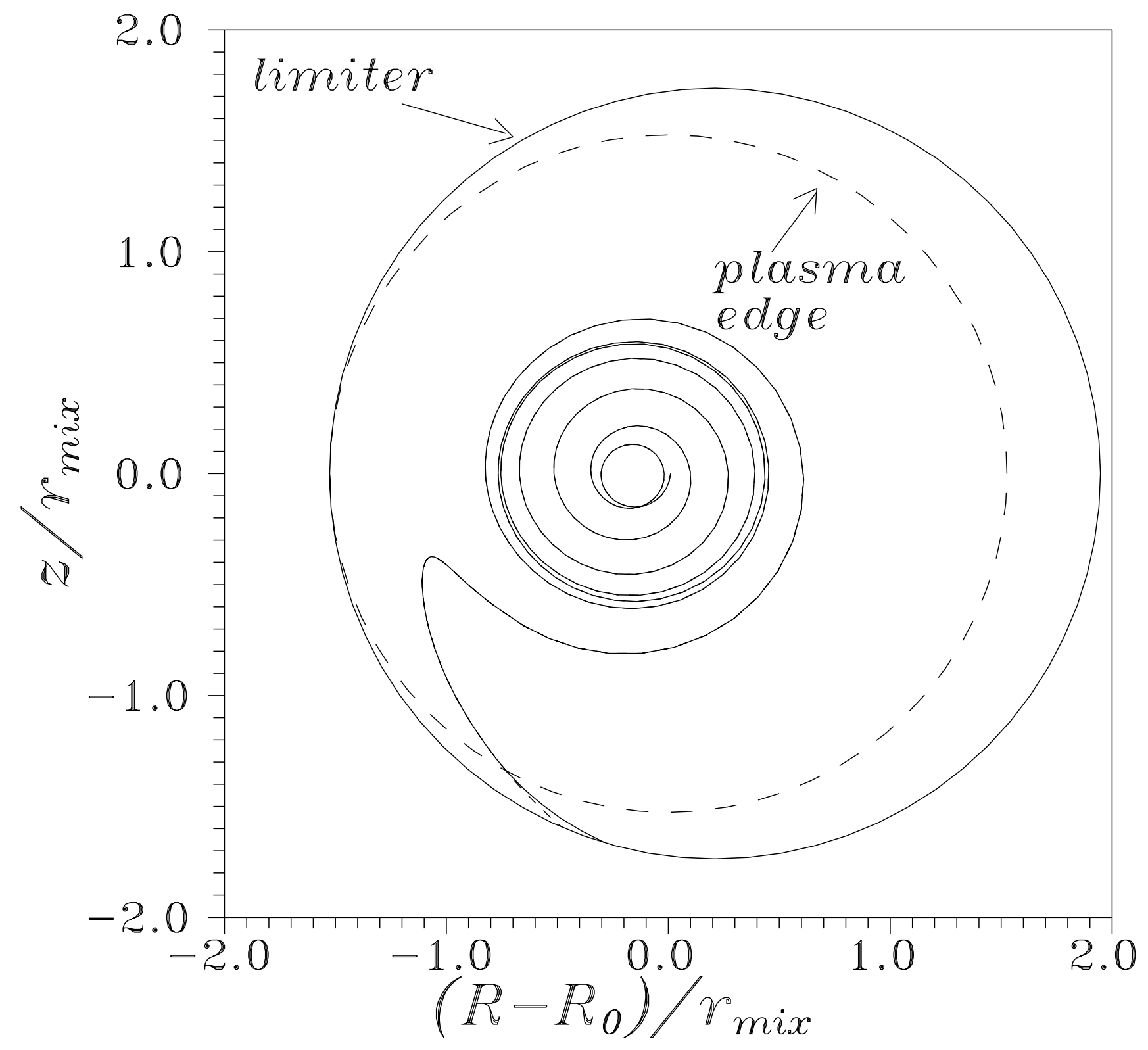

FIG. 5c. 


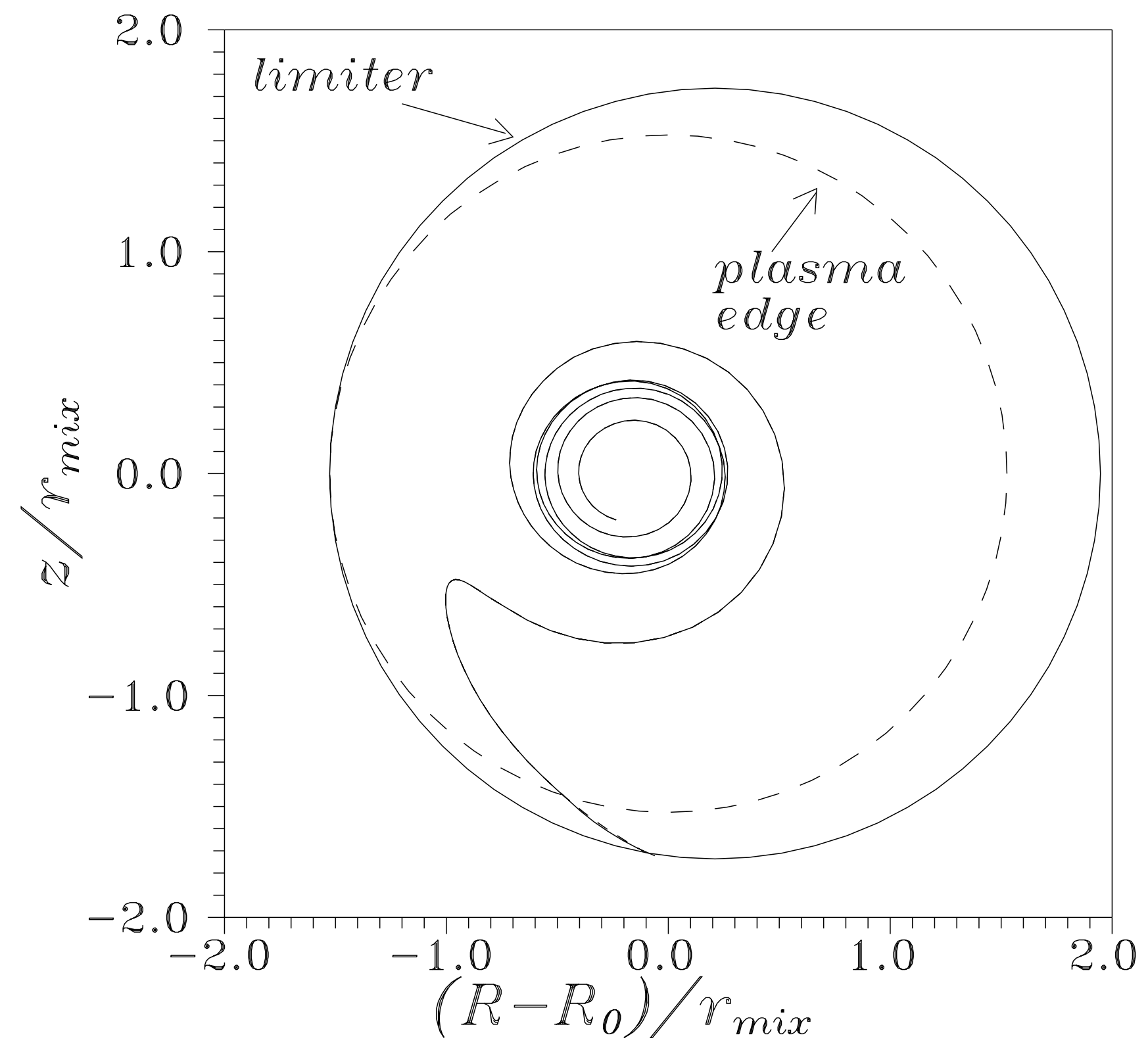

FIG. 5d. 


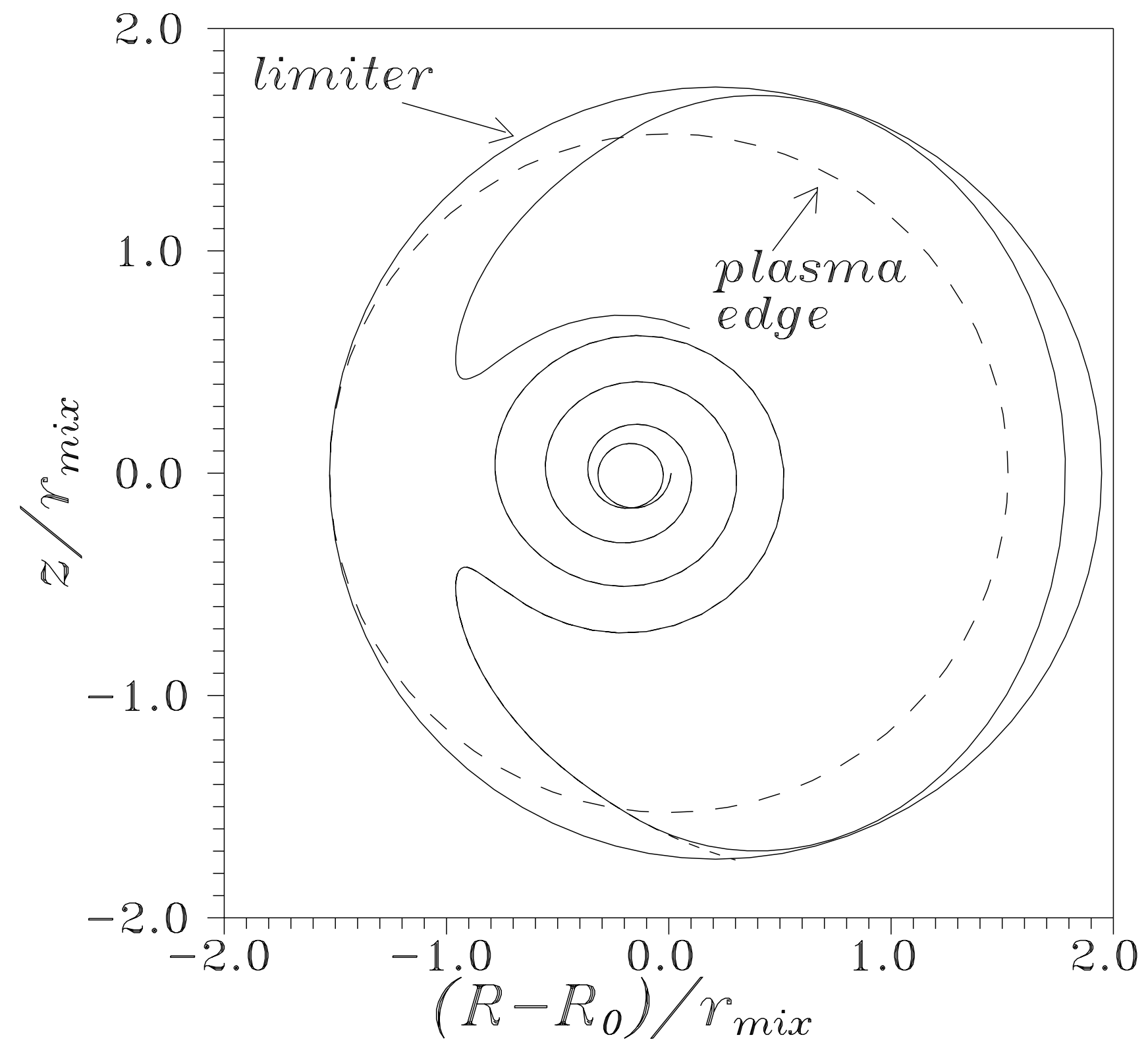

FIG. 5e. 


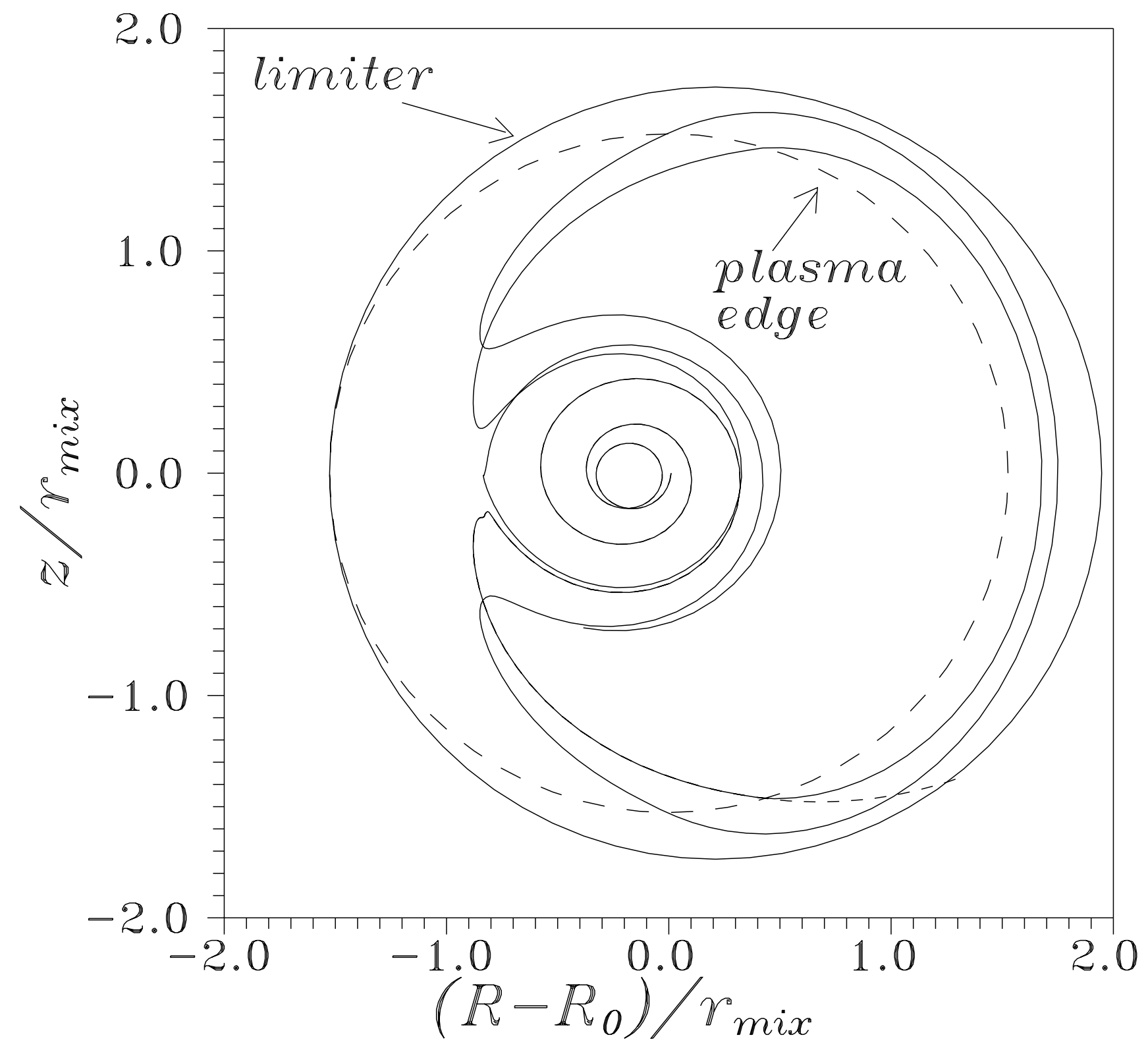

FIG. 5f. 


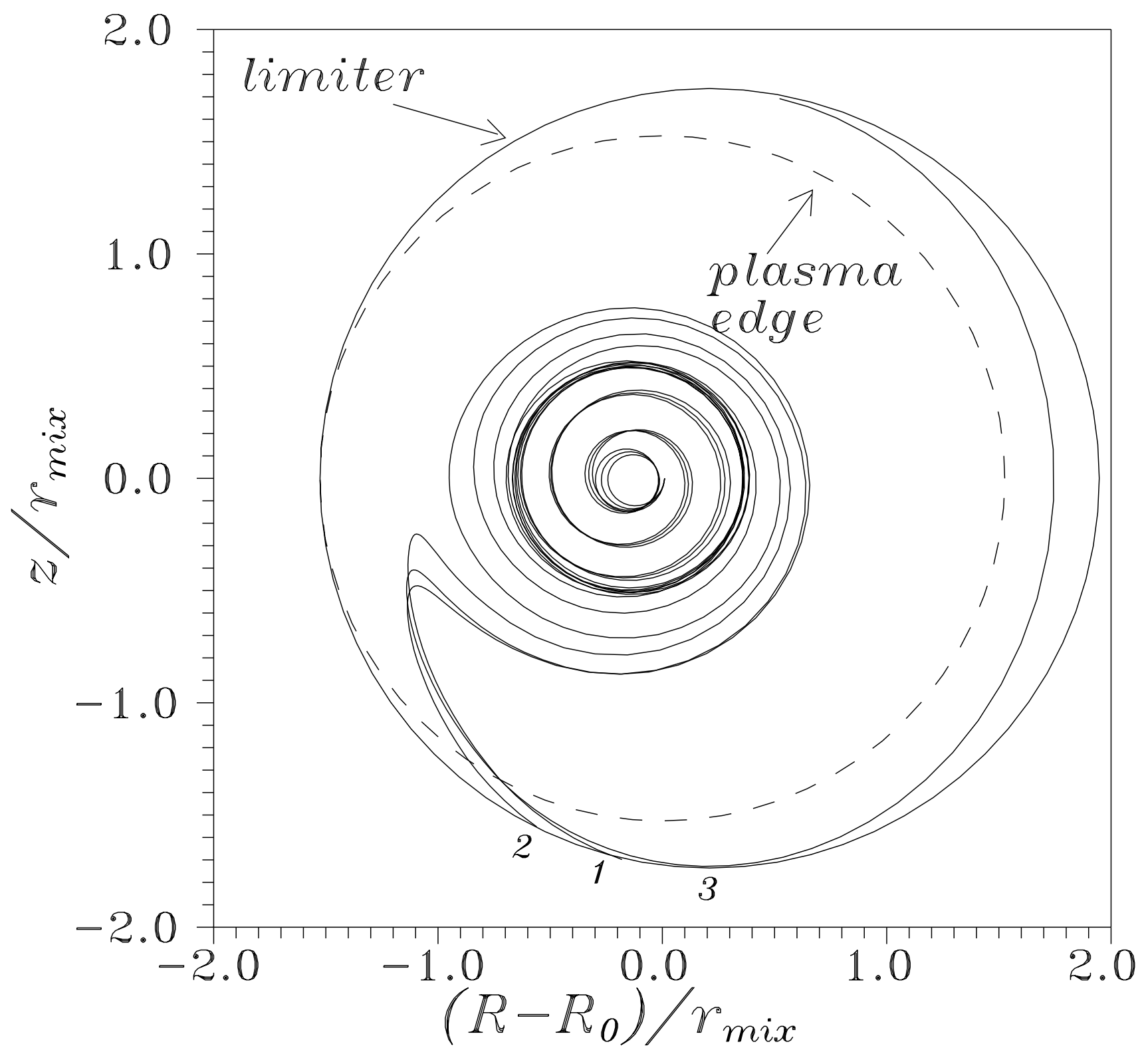

FIG. 6. 


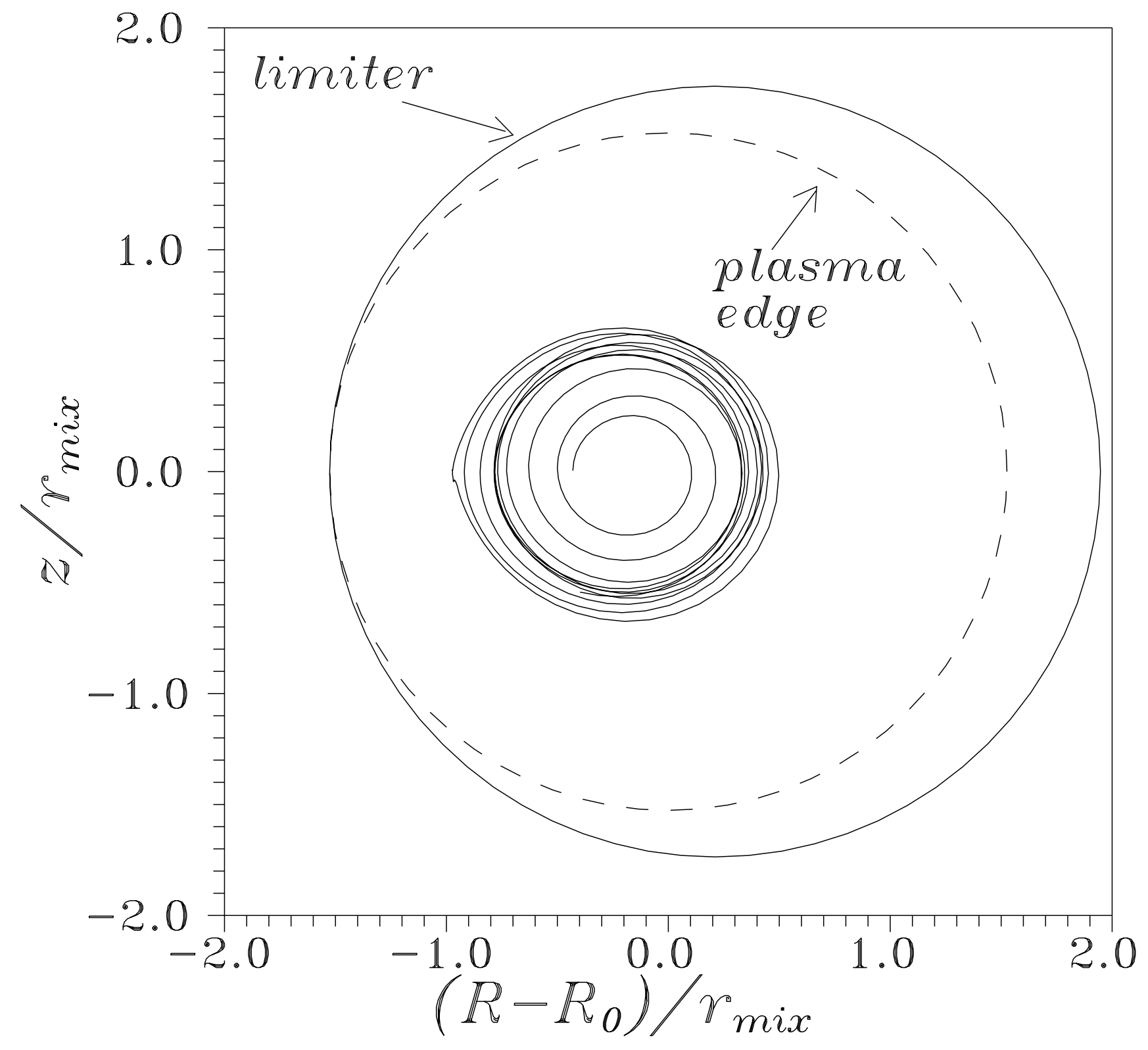

FIG. 7. 


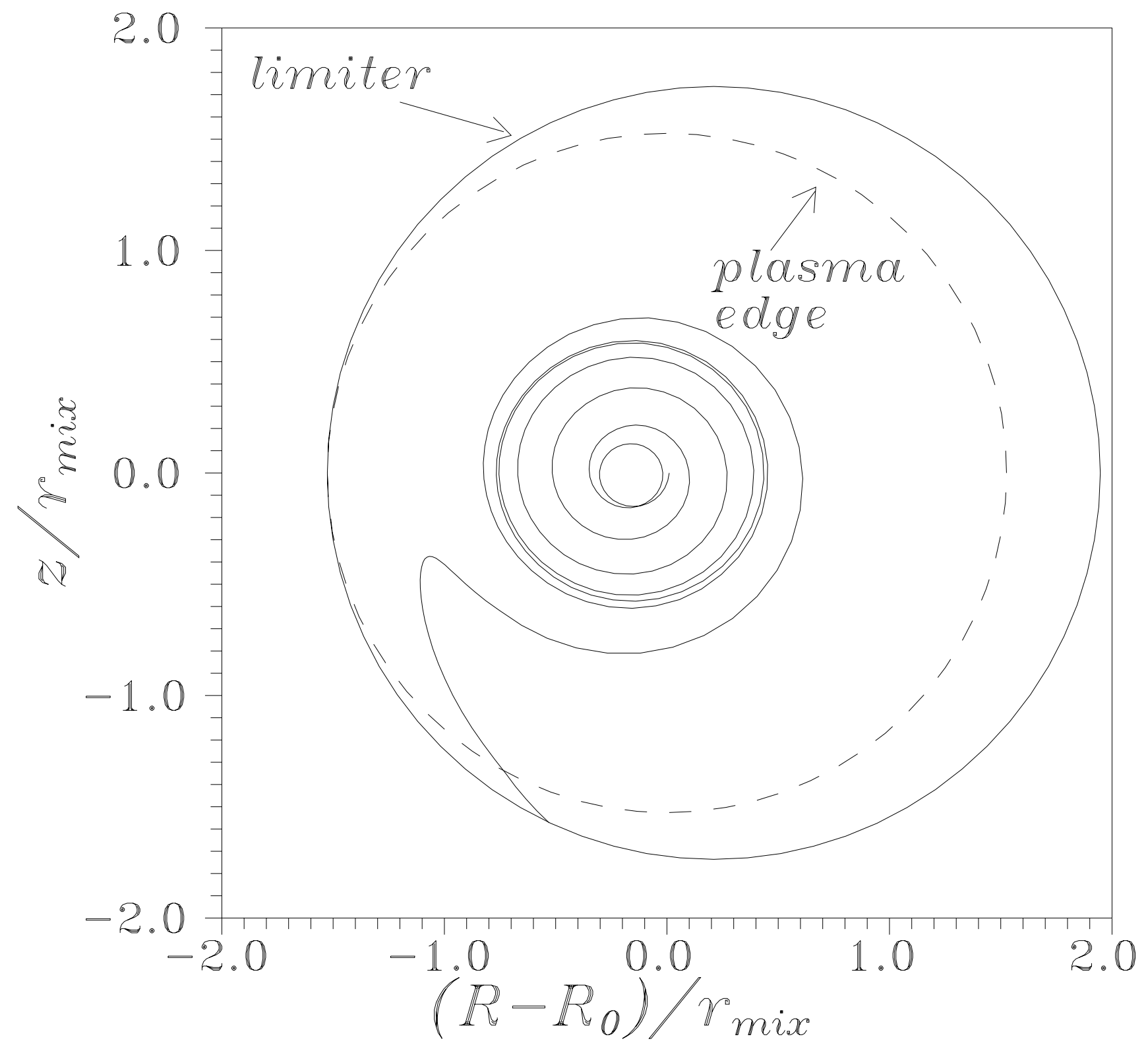

FIG. 8a. 


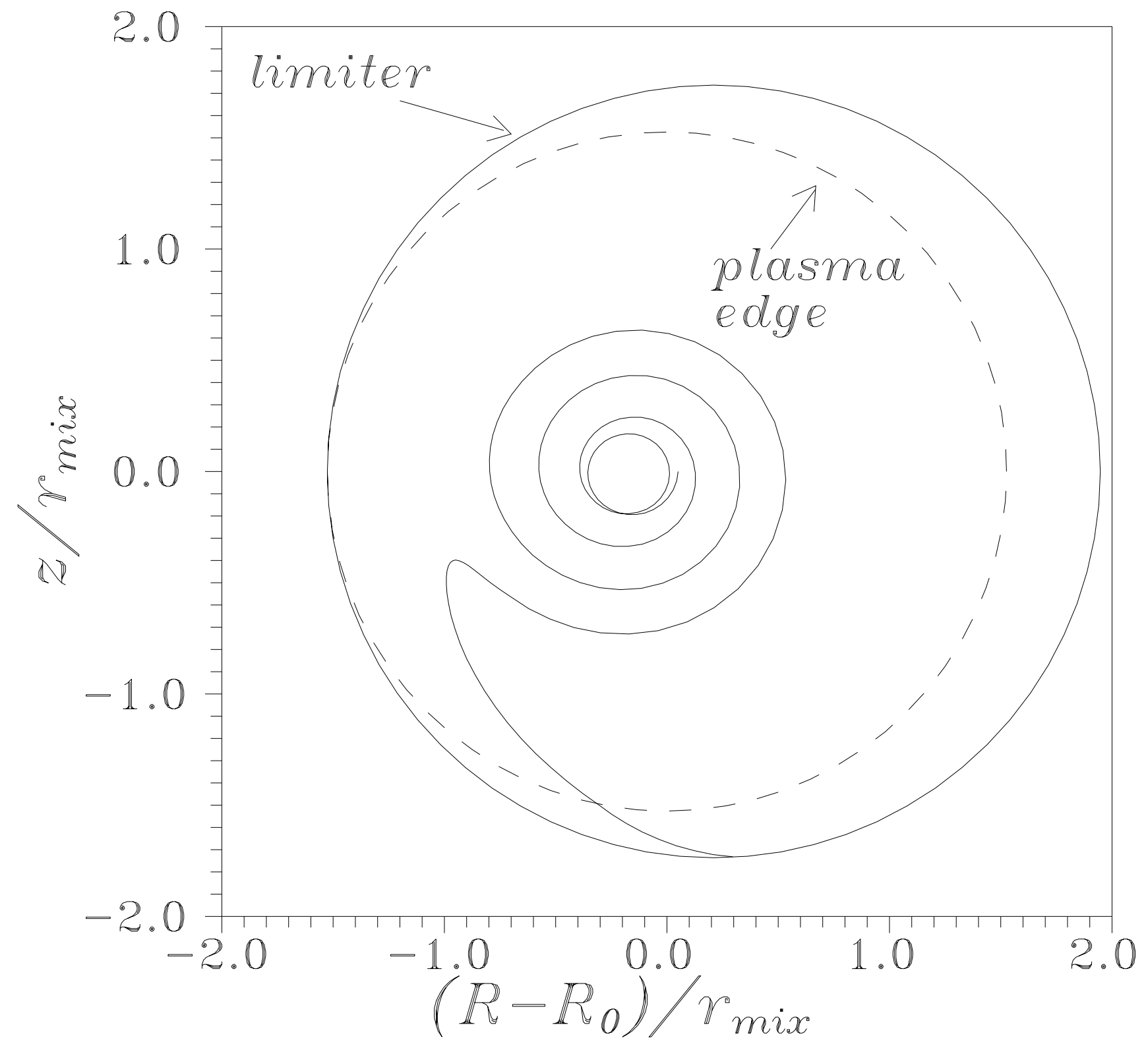

FIG. 8b. 


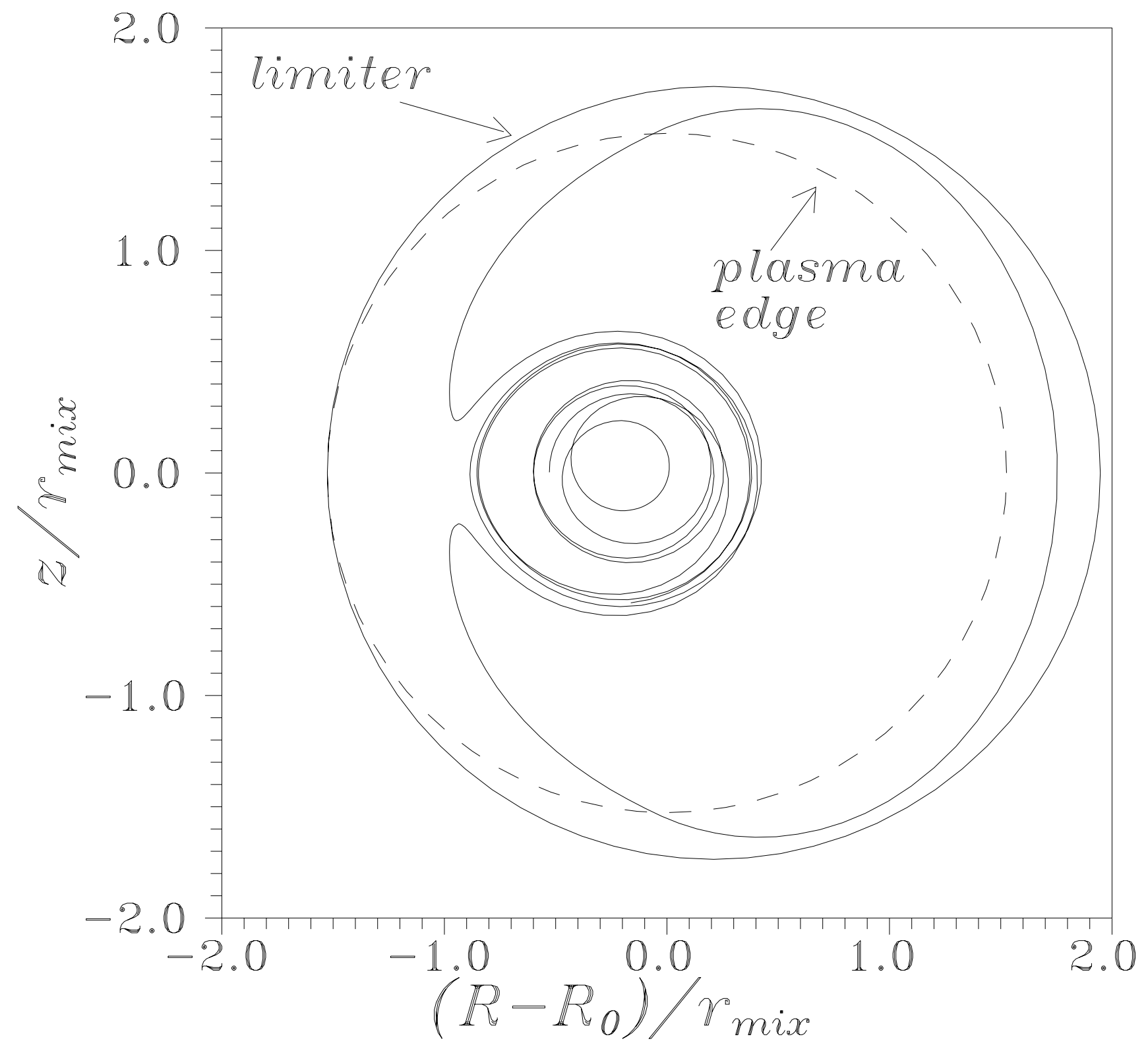

FIG. 8c. 


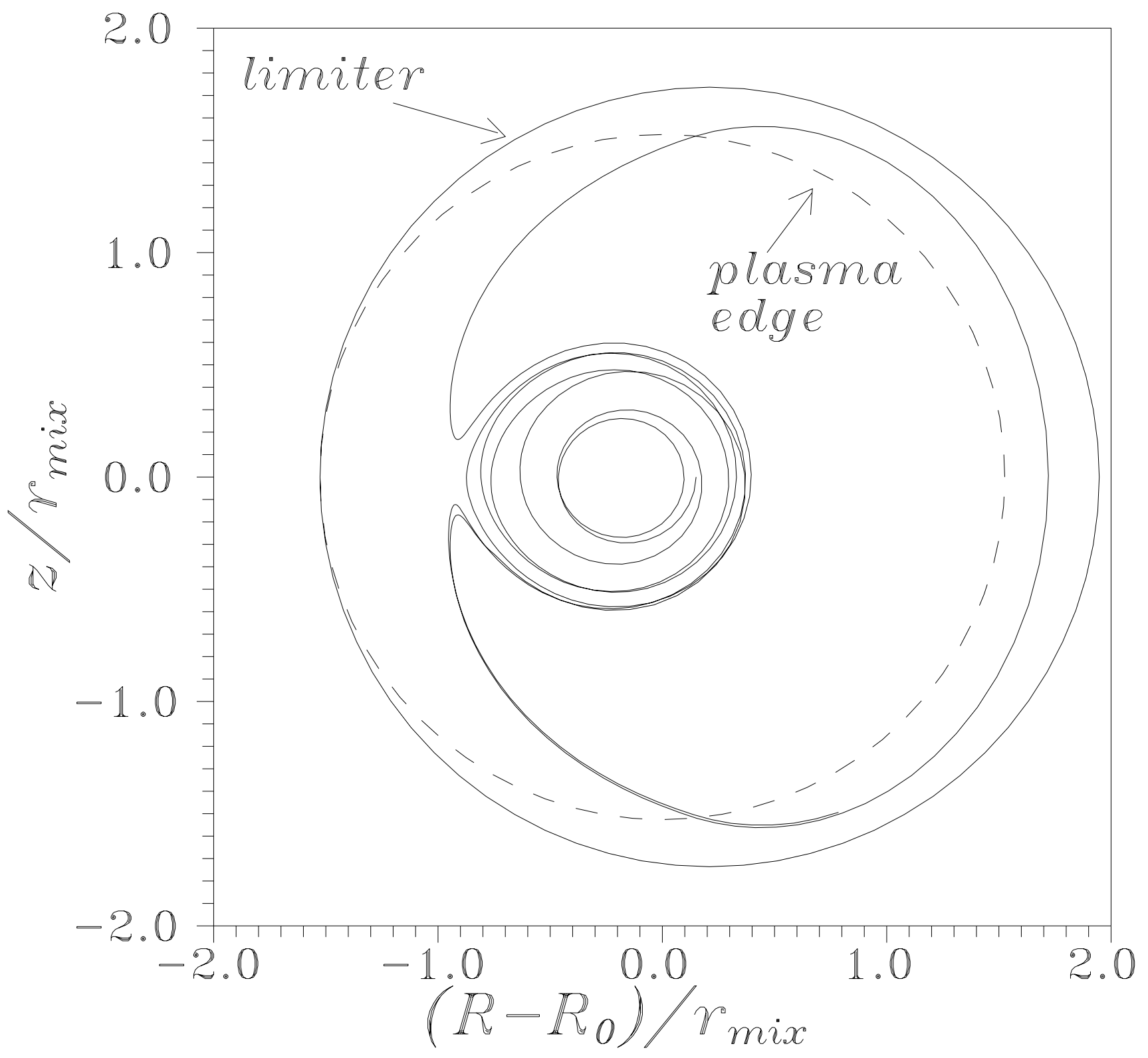

FIG. 8d. 


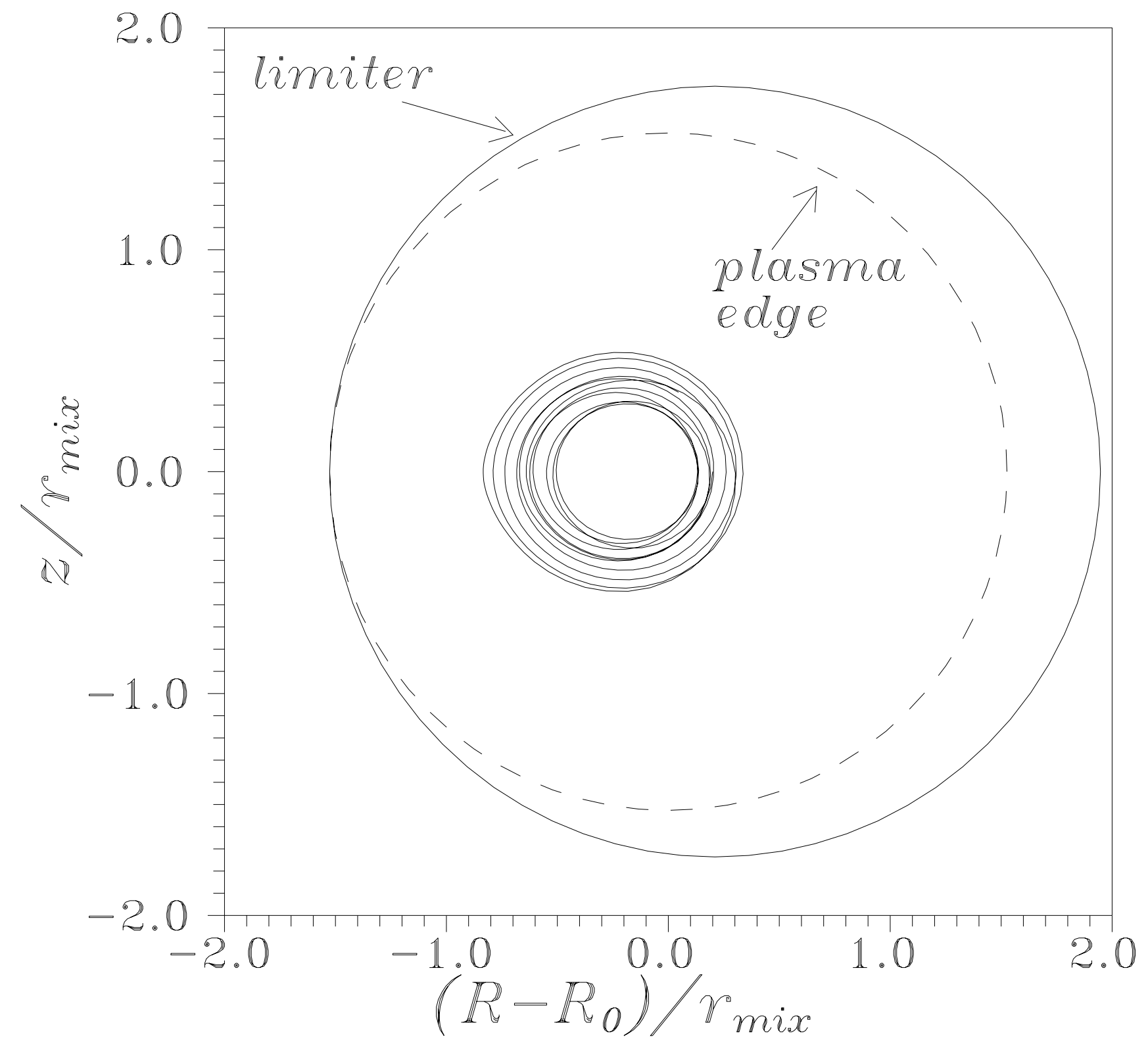

FIG. 8e. 


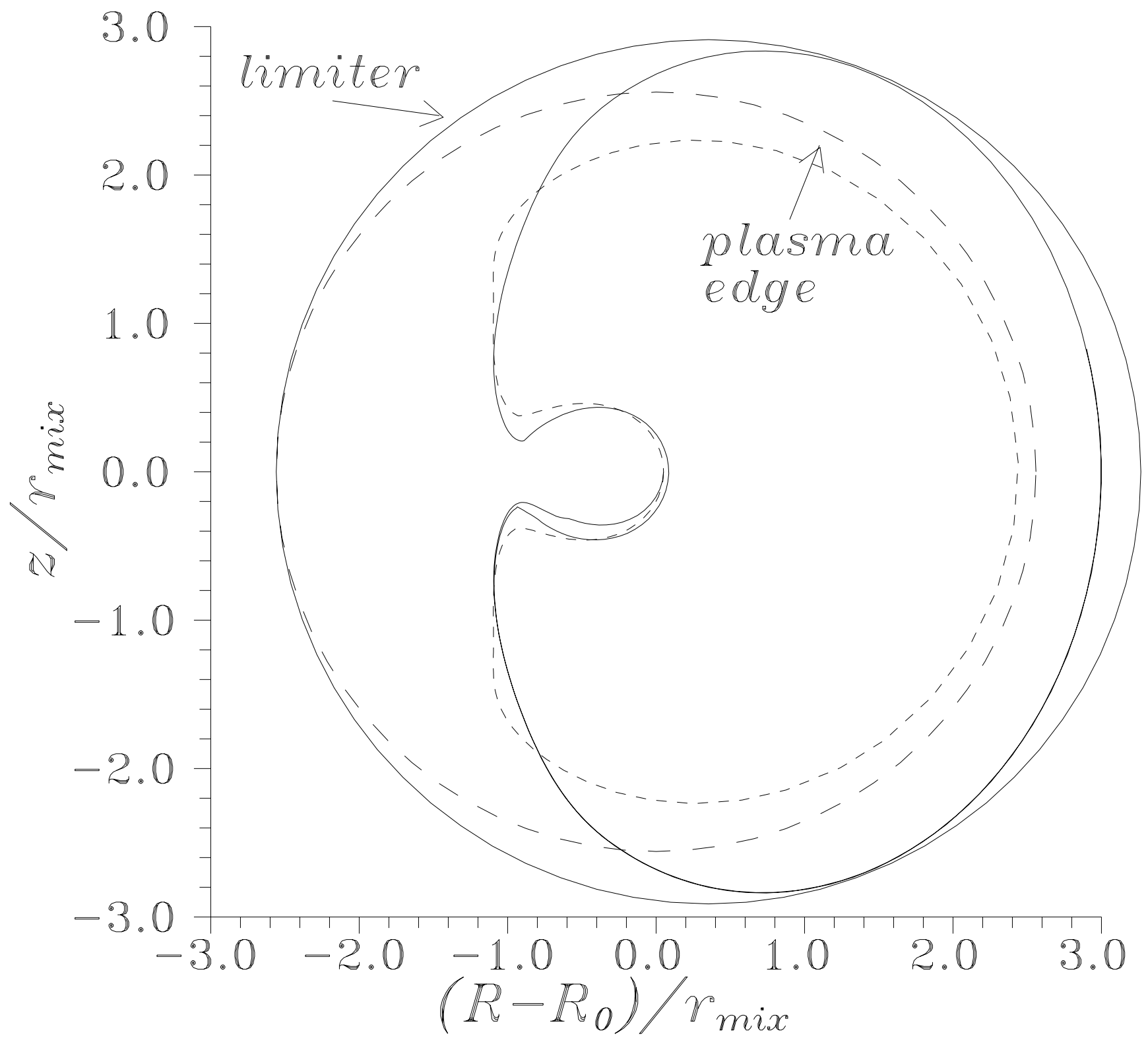

FIG. 9. 


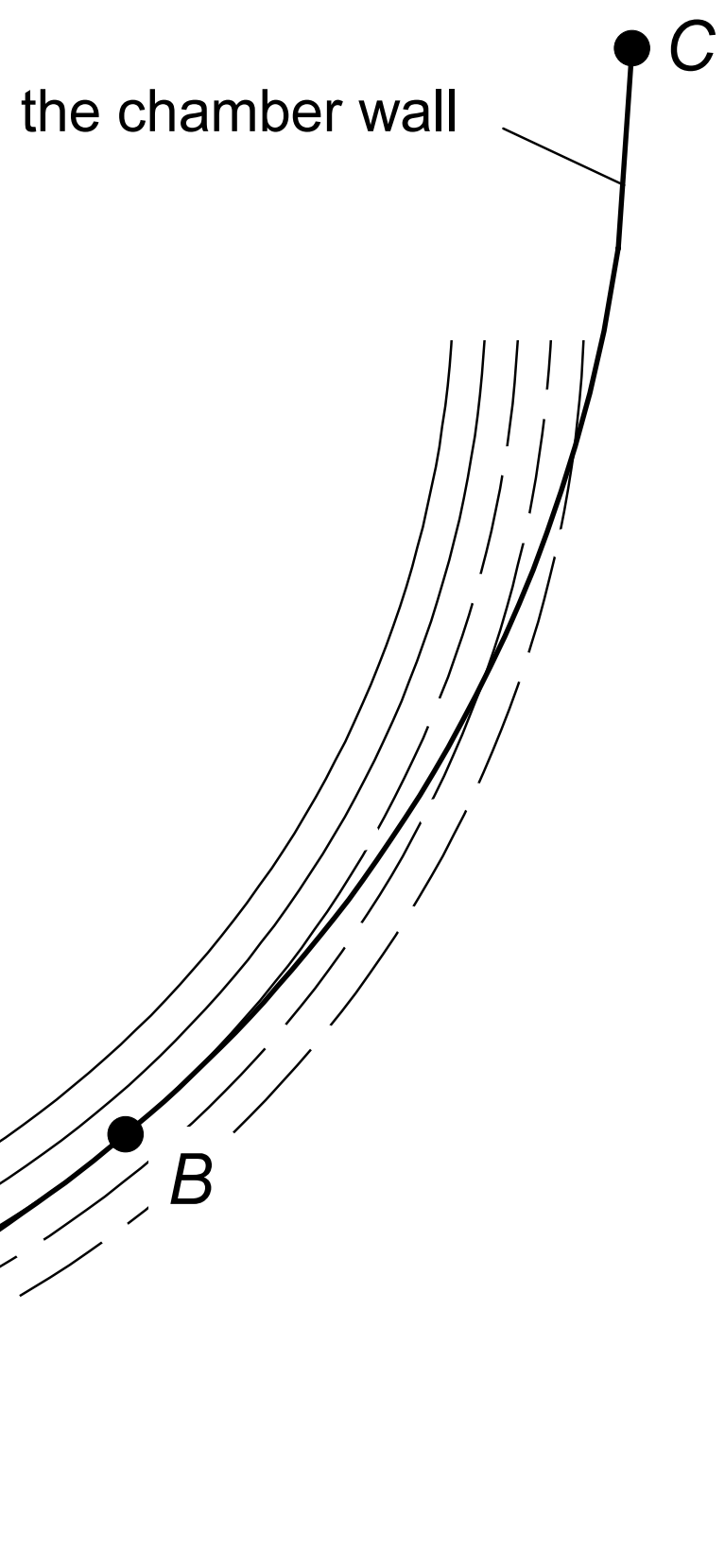

FIG. 10. 


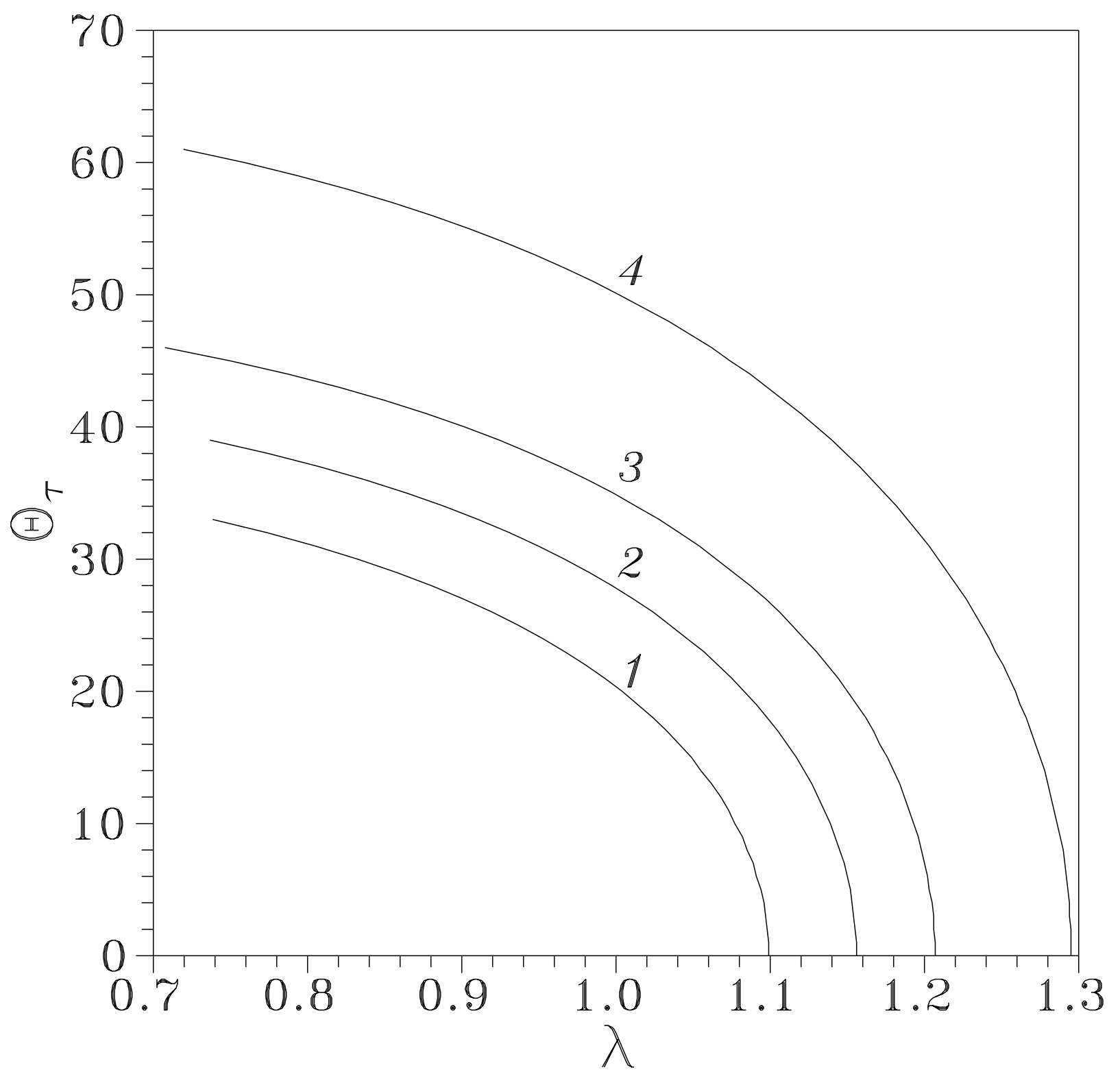

FIG. 11. 

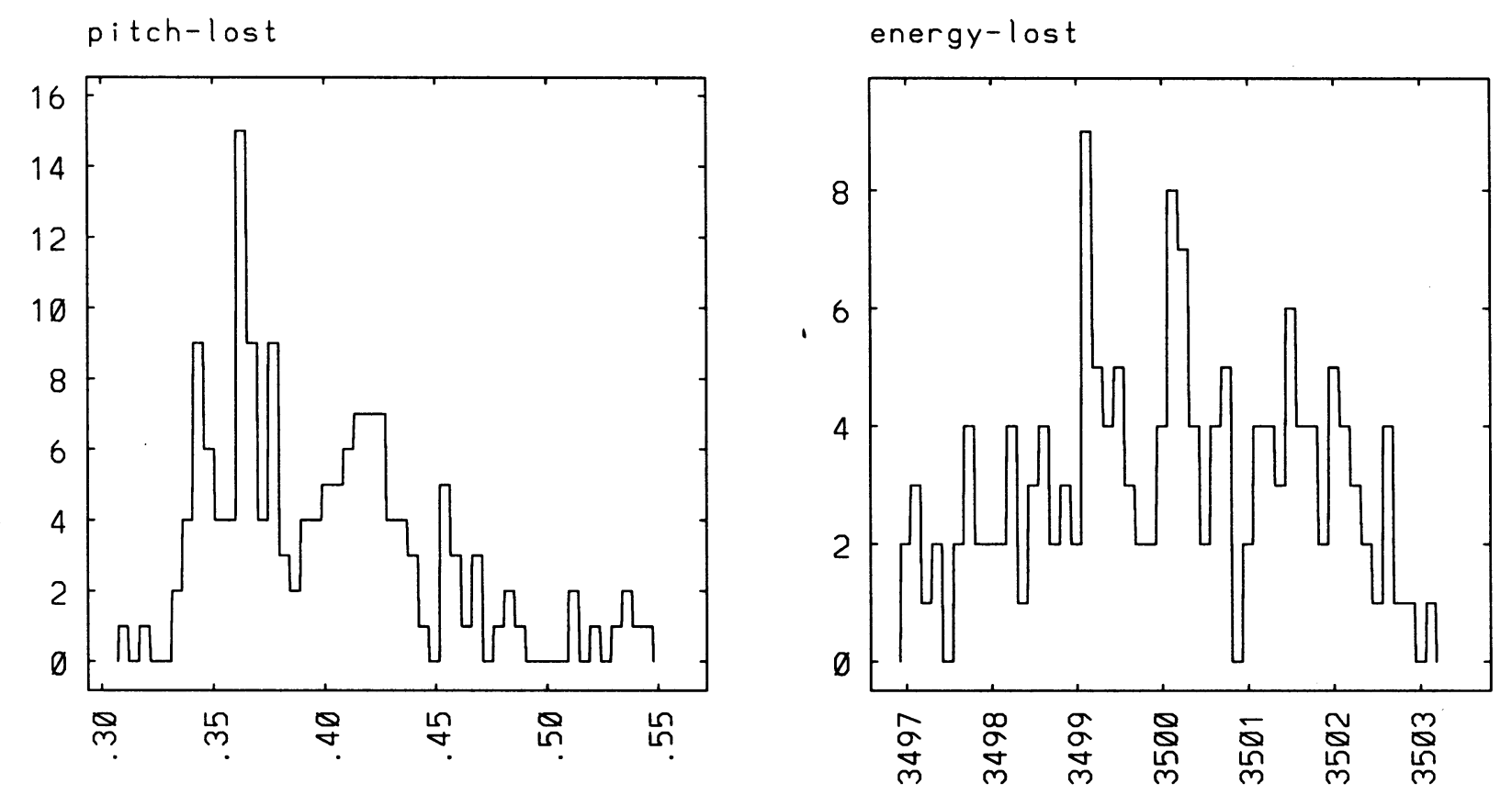

FIG. 12. 


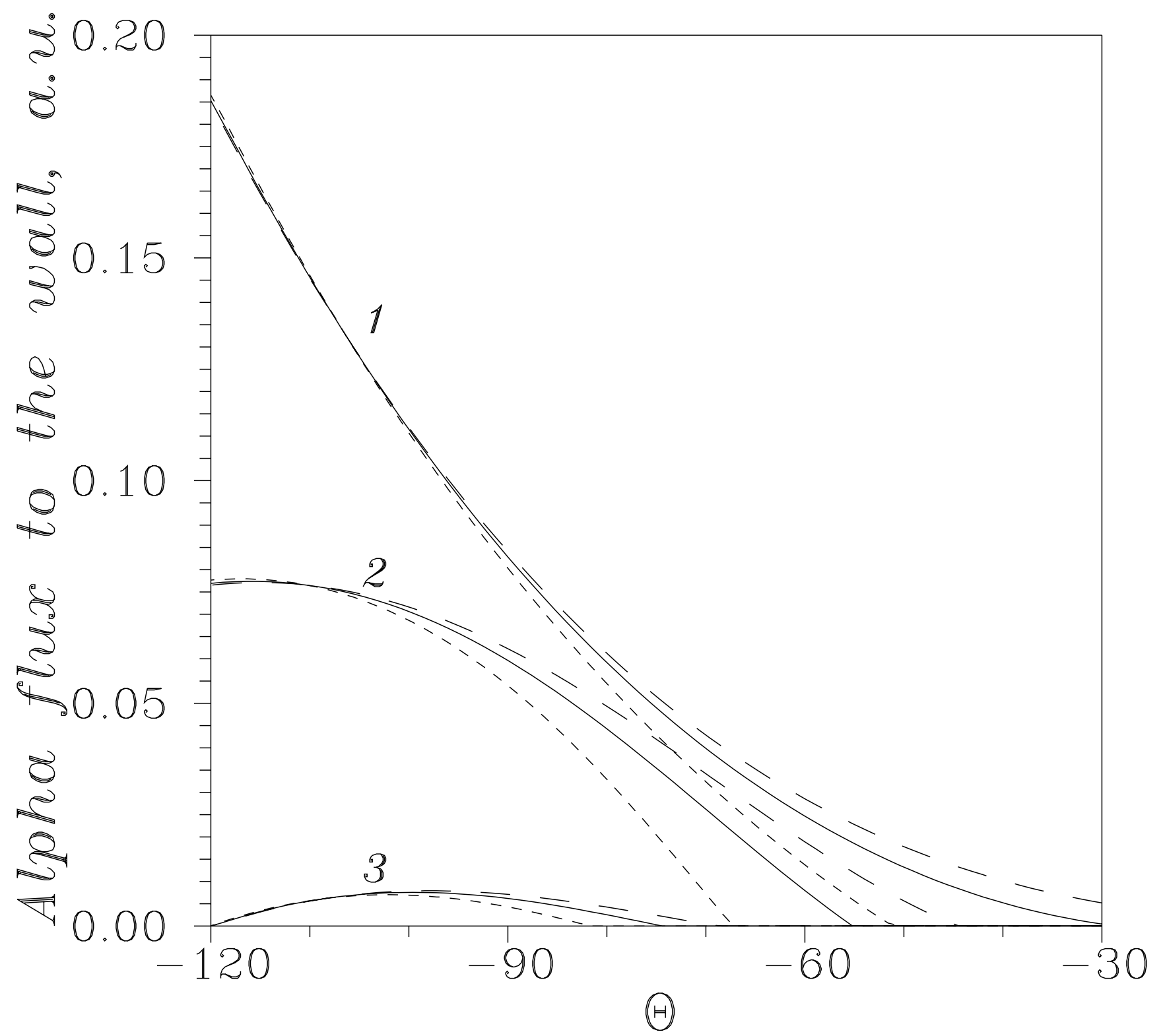

FIG. 13. 


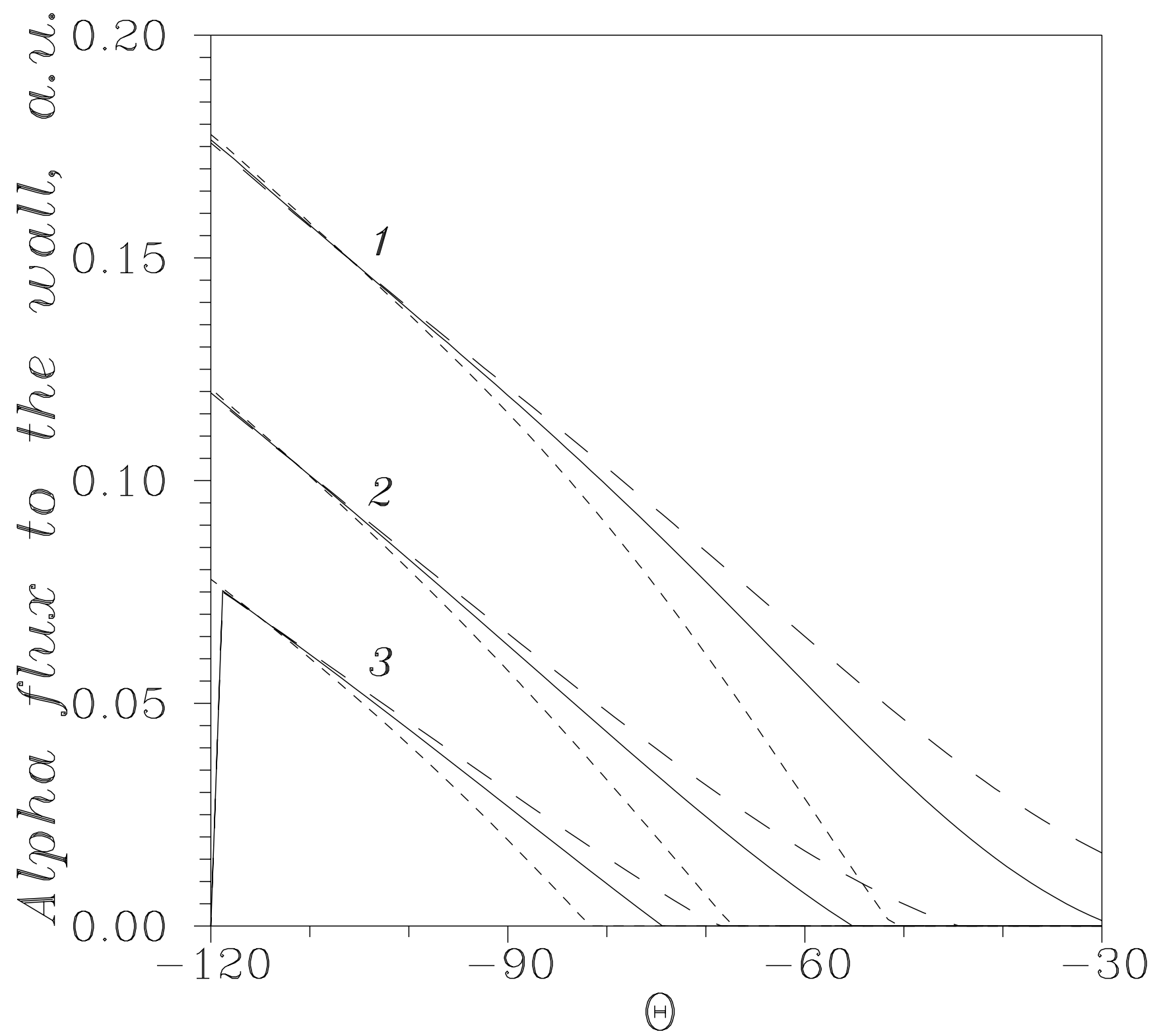

FIG. 14. 


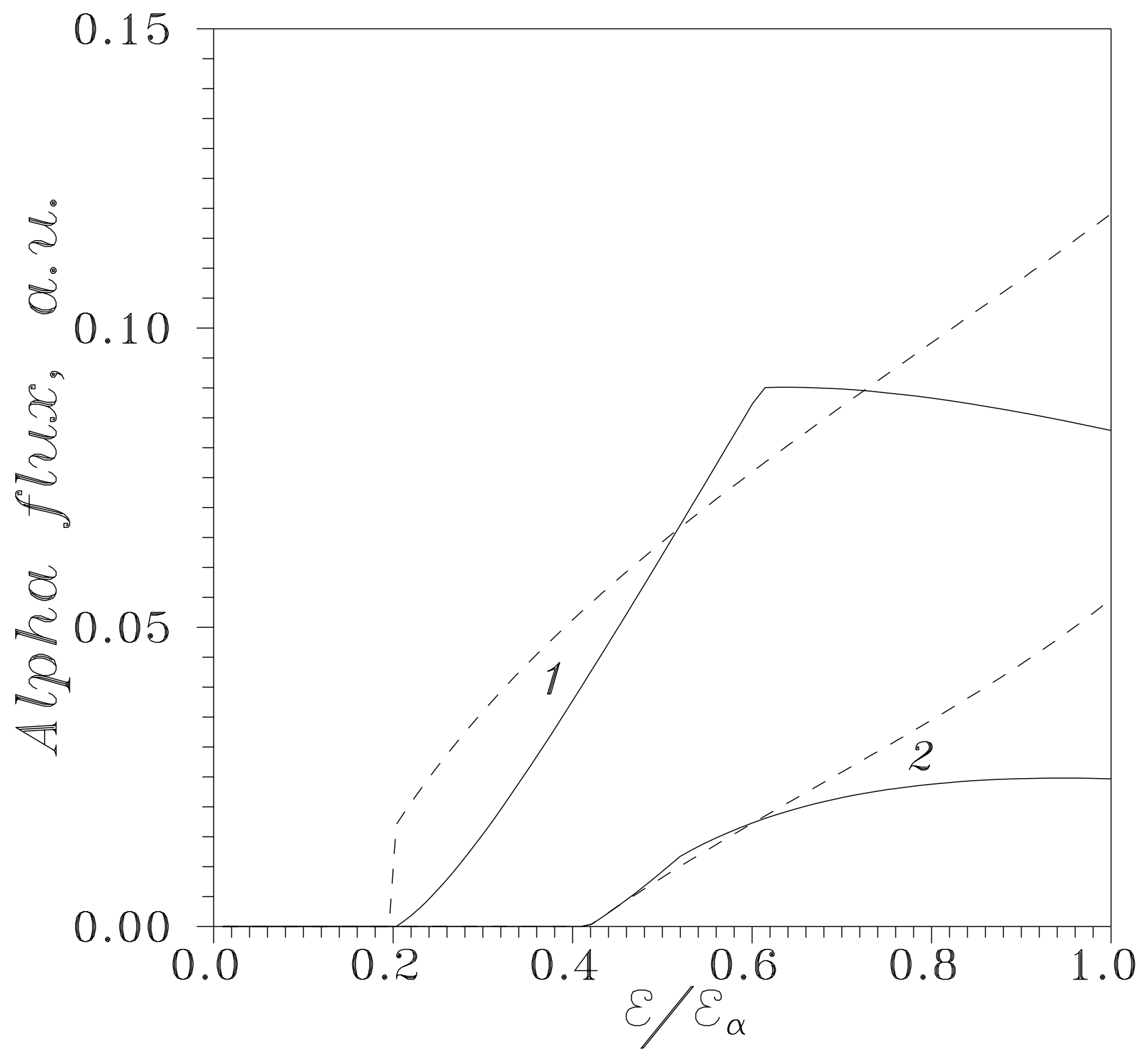

FIG. 15. 


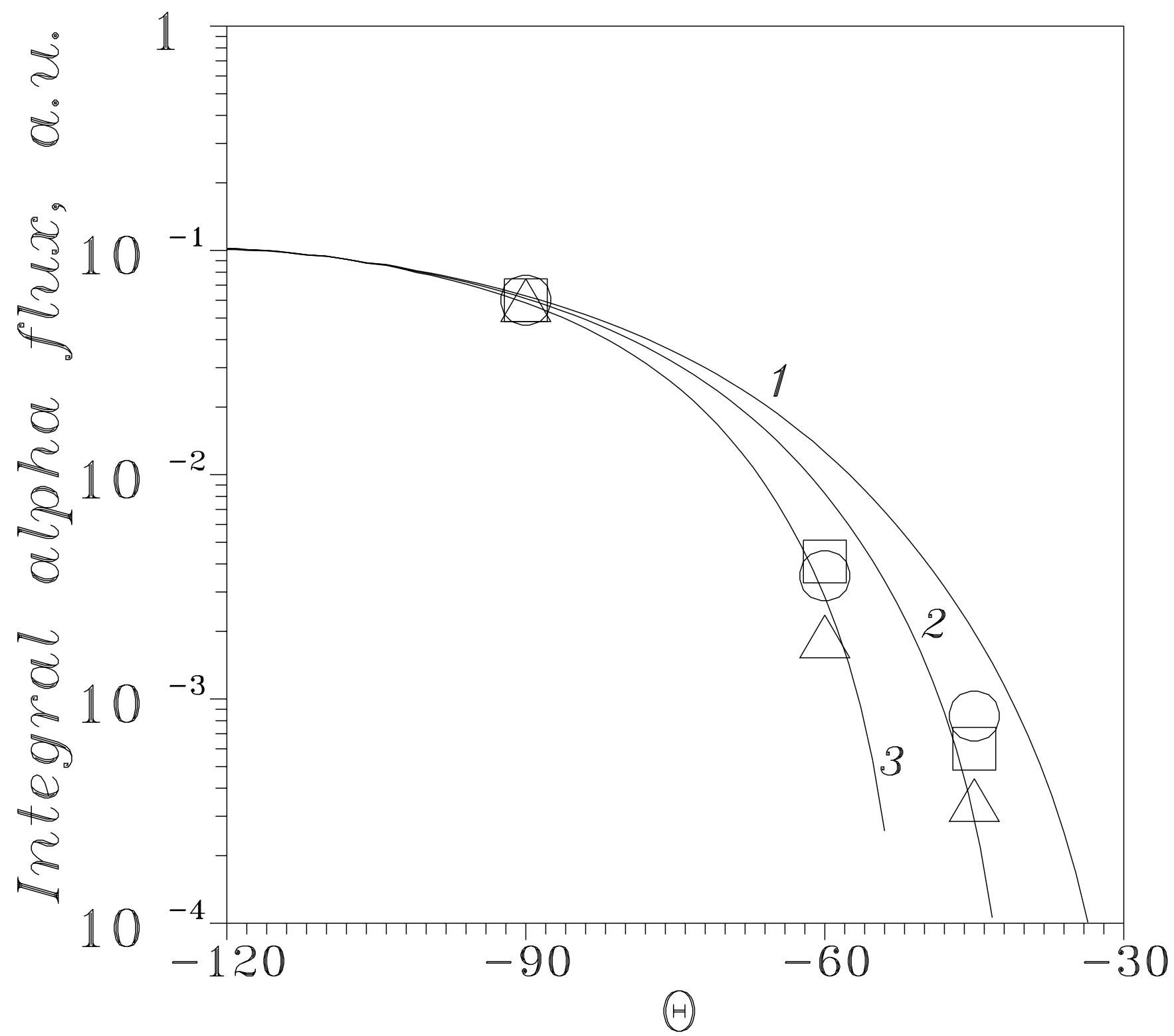

FIG. 16. 


\section{List of Figures}

FIG. 1. Example of a DT discharge with sawtooth-induced alpha loss $\Gamma$ with $I=1.4 \mathrm{MA} \Gamma$ $R=2.52 \mathrm{~m}$ Tand $P_{N B I}=7.5 \mathrm{MW}$ from 4.0 to $4.7 \mathrm{~s}$. Each sudden drop in the central electron temperature is a sawtooth crash. Note that in this case only two of these sawteeth caused alpha loss.

FIG. 2. Time dependence of the alpha loss during the sawtooth crash at $4.7 \mathrm{~s}$ in the discharge of Fig. 1. The drop in the central electron temperature occurs within an $\approx 50 \mu$ s time period near 4.699 s $\Gamma$ and the increase in alpha loss occurs over an $\approx 200 \mu$ s time period. Each of the three detectors which measured the sawtooth-induced alpha loss had a slightly different time dependence.

FIG. 3. Example of the poloidal distribution of the sawtooth-induced alpha loss in a discharge similar to that shown in Fig. 1 . The alpha loss is largest in the $90^{\circ}$ detector and not visible in the $45^{\circ}$ detector. The sampling rate is only $5 \mathrm{kHz}$ for these signals. The different detector sensitivities are not taken into account in the vertical scales $\Gamma$ and each signal has a slowly varying neutron/gamma background which was not subtracted.

FIG. 4. Schematic view of the cross section of the TFTR vacuum vessel.

FIG. 5. The effect of a sawtooth crash on alpha particles with various $\lambda$. (a) $\Gamma \lambda=0.736$; (b) $\Gamma \lambda=0.74 ;(\mathrm{c}) \Gamma \lambda=0.76 ;(\mathrm{d}) \Gamma \lambda=0.78 ;(\mathrm{e}) \Gamma \lambda=0.788 ;$ (f) $\Gamma \lambda=0.8$.

FIG. 6. The effect of a sawtooth crash on alpha particles with various energies. $1 \Gamma 3.5 \mathrm{MeV}$; $2 \Gamma 3 \mathrm{MeV} ; 3 \Gamma 2.5 \mathrm{MeV}$.

FIG. 7. Behavior of a particle with the same parameters as in Fig. 5 (d) but with a different initial phase with respect to the pertubation.

FIG. 8. Effect of a sawtooth crash on particles with various pre-crash radial position.

FIG. 9. Crash-induced loss of an "exotic" trapped particle.

FIG. 10. Sketch of particle orbits with $v_{\|}>0$ near the chamber wall. Particles cannot reach the parts of the orbits drawn by dashed lines Tas well as the part of the wall marked as $B C$. FIG. 11. $\theta_{\tau}$ versus $\lambda$ for various values of $\mathcal{E}$ in the shot $\# 87530.1 \Gamma \mathcal{E}=3.5 \mathrm{MeV} ; 2 \Gamma$ 
$\mathcal{E}=3.0 \mathrm{MeV} ; 3 \Gamma \mathcal{E}=2.5 \mathrm{MeV} ; 4 \Gamma \mathcal{E}=1.5 \mathrm{MeV}$

FIG. 12. The pitch-angle and energy distribution of the alpha ripple loss for a source function simulating a crash.

FIG. 13. The poloidal distribution of the alpha prompt loss induced by the Kadomtsev-type crash for various particle energy and Shafranov shift. $1 \Gamma \mathcal{E}=3.5 \mathrm{MeV} ; 2 \Gamma \mathcal{E}=1.75 \mathrm{MeV} ; 3 \Gamma$ $\mathcal{E}=0.87 \mathrm{MeV} ;$ solid line $\Gamma \Delta^{\prime}=1.67 A^{-1} ;$ dashed line $\Gamma \Delta^{\prime}=A^{-1} ; \operatorname{dotted} \operatorname{line} \Gamma \Delta^{\prime}=2 A^{-1}$.

FIG. 14 The same as Fig. 13 but for $V\left(r_{X}\right)=$ const.

FIG. 15. The energy spectrum of the sawtooth-induced alpha prompt loss. $1 \Gamma$ the $90^{\circ}$ detector; $2 \Gamma$ the $60^{\circ}$ detector; solid line $\Gamma$ the Kadomtsev-type crash; dotted line $\Gamma V\left(r_{X}\right)=$ const.

FIG. 16. The poloidal distribution of the integral alpha flux induced by the Kadomtsevtype crash for various Shafranov shift in the TFTR shot \#87530. Solid lines $\mathrm{T}$ results of calculations; $1 \Gamma \Delta^{\prime}=1.67 A^{-1} ; 2 \Gamma \Delta^{\prime}=1.8 A^{-1} ; 3 \Gamma \Delta^{\prime}=2 A^{-1}$; symbols $\Gamma$ experimental points fot three crashes. 